\title{
Magnetoelastic deformation of a circular membrane: wrinkling and limit point instabilities
}

\author{
Prashant Saxena ${ }^{1,2 *}$ Narravula Harshavardhan Reddy ${ }^{3}$, \\ Satya Prakash Pradhan ${ }^{2}$ \\ ${ }^{1}$ Glasgow Computational Engineering Centre, James Watt School of Engineering \\ University of Glasgow, Glasgow G12 8LT, UK. \\ ${ }^{2}$ Department of Mechanical \& Aerospace Engineering, Indian Institute of Technology \\ Hyderabad, Kandi, Sangareddy 502285, Telangana, India. \\ ${ }^{3}$ Graduate Aerospace Laboratories, California Institute of Technology, \\ Pasadena 91125, California, USA.
}

\begin{abstract}
We study the inflation of a weakly magnetizable isotropic incompressible circular membrane in the presence of magnetic field generated by a magnetic dipole. Following the approach in recent papers by (Reddy and Saxena 2018, Int. J. Sol. Struct. 136, 203-219; Reddy and Saxena 2017, Int. J. Non-Lin. Mech. 95, 248-263) we start with a variational formulation, solving the resulting governing equations to determine the equilibria and checking the second variation condition for stability. Conjecture of possibility of multiple equilibria under a single coupled load, and attaining elastic and magnetic limit points made in the above two papers is confirmed in the present work for a circular membrane. Another main focus of this work is on the determination of wrinkling instability in the membrane due to magnetoelastic stresses. Wrinkles along one or both in-plane directions of membrane appear in a majority of loading scenarios due to compressive Maxwell stresses. Our computations demonstrate that wrinkles arise in the central region when dipole and inflation are in the same direction and in the annular region close to the edges when the dipole and inflation are in opposite directions.
\end{abstract}

Keywords: magnetoelasticity; wrinkling; instability; membrane; limit point

Note: This is an author generated version of the article accepted for publication in the "International Journal of Nonlinear Mechanics" on 12 July 2019.

\section{Contents}

1 Introduction

2 Kinematics of deformation 3

2.1 Problem description . . . . . . . . . . . . . . . . . . . . . . 3

2.2 Reference Configuration . . . . . . . . . . . . . . . . . . . 4

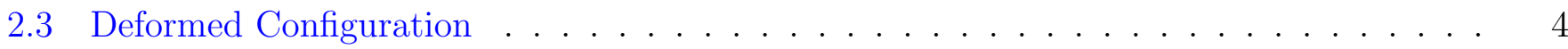

${ }^{*}$ Corresponding author. Email: prashant.saxena@glasgow.ac.uk, Phone: +44 1413302000 Ext: 0927 
3.1 Total potential energy and its variation . . . . . . . . . . . . . . . 6

3.1 .1 Energy due to magnetic field of dipole . . . . . . . . . . . . . . . 7

3.1.2 Constitutive model for the elastic strain energy . . . . . . . . . . . . . . 8

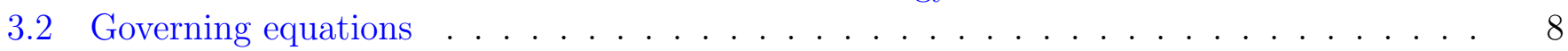

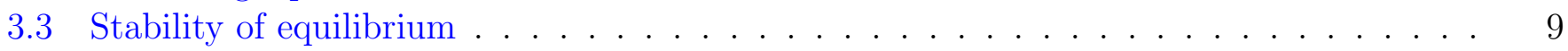

3.4 Magnetoelastic stresses and wrinkling . . . . . . . . . . . . . . . . . . 10

3.5 Numerical solution procedure . . . . . . . . . . . . . . . . . . . . . . . 11

4 Numerical results $\quad 12$

4.1 Validating the formulation . . . . . . . . . . . . . . . . . . . . . . . . . . . . . . . . . . . . .

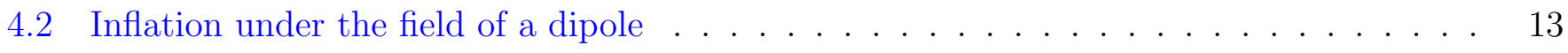

4.2.1 Pressure-volume characteristics and limit points . . . . . . . . . . . . . 13

4.2 .2 Wrinkling behaviour . . . . . . . . . . . . . . . . . 15

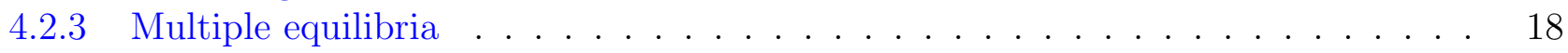

\section{Conclusions}

A Calculation of matrices $\mathrm{P}$ and $\mathrm{Q}$

\section{Introduction}

Magnetoelastic polymers are smart materials that demonstrate a change of mechanical properties under the influence of an external magnetic field. A common manufacturing technique involves dispersion of micron-sized ferromagnetic particles in a polymer matrix and then curing the mixture (Jolly et al., 1996). Magnetization of these particles manifests itself as a change in macroscopic properties such as stiffness or dimensions of the composite (Böse and Röder, 2009). This phenomenon is utilized in several engineering applications including flexible robotics (Hu et al., 2018), active vibration and stiffness control (Ginder et al., 2001, 2002; Mayer et al., 2013), waveguides (Saxena, 2017), and actuators (Böse et al., 2012).

Early theoretical studies on magnetoactive materials have been around for more than half a century. Some prominent works include those by Truesdell and Toupin (1960); Tiersten (1964); Brown (1966) and then later by Maugin and Eringen (1972); Pao (1978). Using the direct approach (based on balance laws for continua) or a variational formulation based approach, similar governing equations are arrived at. Interest in computational and mathematical modelling of magnetoelasticity has been revived in recent years due to the engineering developments mentioned in the previous paragraph. Based on these classical texts, Dorfmann and Ogden (2003), Kankanala and Triantafyllidis (2004), and Steigmann (2004) developed theories of coupled nonlinear magnetoelasticity to model interactions between magnetic field and finitely deformed solids. The total free energy density is expressed as a function of the deformation gradient and one variable among the magnetic flux density, field intensity, or magnetization vector. Several advanced techniques have been proposed to model additional features of the particle-filled magnetoelastic smart elastomers. For example, Castañeda and Galipeau (2011); Castaneda and Siboni (2012) and Chatzigeorgiou et al. (2014) formulated the field equations by coupling magneto-mechanical phenomena at the microscopic level and the use of homogenization; Saxena et al. (2013); Ethiraj and Miehe (2016) and Haldar et al. (2016) modelled the energy dissipation due to coupled polymer magneto-viscoelasticity; Bustamante (2010); Danas et al. (2012) and Saxena et al. $(2014,2015)$ modelled the inherent anisotropy and resulting changes in the magneto-mechanical response of these polymers. Several of the above-mentioned models have been useful in computational analysis of instabilities in magnetoelastic bulk media reported by Otténio et al. (2008); Kankanala and 
Triantafyllidis (2008); Rudykh and Bertoldi (2013); Danas and Triantafyllidis (2014); Saxena (2017), and Goshkoderia and Rudykh (2017).

Nonlinear elastic membranes find applications in areas such as inflatable space-systems (Grossman, 1991a,b), blow moulding (Khayat and Derdouri, 1994a,b), and bio-medical equipments (Leone, 1994) to name a few. In the regime of large deformations, limit point (snap-through) instability is an important bifurcation phenomenon occurring in elastic membranes. Internal pressure required to stretch the membrane rises with the deformation until the limit point and then drops causing a sudden increase in the membrane dimensions ( knowledge of this critical pressure is crucial to maintain the deformation of membranes within acceptable limits. In the context of membranes under coupled loading, Rudykh et al. (2012) studied the snapthrough actuation of spherical dielectric membranes while Liang and Cai (2017) studied the same in circular dielectric membranes. Wrinkling is another form of common bifurcation phenomenon in membranes that is associated with development of compressive stresses. As membranes have very small thickness in one dimension with almost zero bending stiffness, any compressive stress in the in-plane direction results in development of out of plane deformations. Tension field theory based on a relaxed energy density approach developed by Pipkin (1986) and Steigmann (1990) has been very successful in analytical modelling of wrinkles - although a counterpart of this theory to magnetoelasticity is not yet available. Recently Reddy and Saxena (2018) have shown the emergence of wrinkles due to magnetic field in the deformation of cylindrical magnetoelastic membranes.

Very few experimental studies have been conducted on magnetoelastic membranes. Raikher et al. (2008) deformed circular magnetoelastic membranes under a uniform magnetic field created by large electromagnets but without a pressure. They report that the neo-Hookean material model used by them is unable to match the experimentally observed nonlinear regime during finite deformation. A parallel problem of deformation of electroelastic membranes has been experimentally studied by Keplinger et al. (2012); Li et al. (2013) and Liang and Cai (2017). They have shown that limit point can be modified upon the application of an external electric field.

Theoretical framework for bulk magnetoelasticity was extended to magnetoelastic membranes by Steigmann (2004) using a formulation based on magnetic field intensity. This was further simplified by Barham et al. $(2007,2008,2010)$ to study the deformation of a weakly magnetized circular membrane in the presence of a stationary dipole. They reported the existence of a magnetic limit point where the stable and unstable equilibria merge. Using their formulation, Reddy and Saxena $(2017,2018)$ studied the deformation of pressurized toroidal and cylindrical magnetoelastic membranes in the presence of external magnetic field generated by current carrying conductors. Their computations showed the existence of an additional limit point before the traditional elastic limit point and the magnetic limit point reported by Barham et al. They also reported the existence of multiple stable and unstable equilibria for a given set of coupled loading conditions.

In this work, we extend the formulation developed in (Reddy and Saxena, 2017, 2018) to study the magnetoelastic deformation of a pressurized circular membrane. We derive the relevant equations of kinematics in Section 2 and express the configuration of axisymmetrically deformed membrane in terms of two deformation parameters. In Section 3, we derive the governing differential equations and associated boundary conditions of the system by minimizing an appropriate potential functional. We use the three-term Ogden energy density function (Ogden, 1972) to model the elastic deformation and derive the necessary and sufficient conditions to analyse stability of the solution. A criteria based on the total magnetoelastic stress is used to model the onset of wrinkling. Results arising from the numerical solution of these equations are presented in Section 4 and we report our conclusions in Section 5.

\section{Kinematics of deformation}

\subsection{Problem description}

Figure 1 shows the incompressible isotropic nonlinear magnetoelastic circular membrane in its reference and deformed configurations. It has a radius $R_{0}$ in the reference configuration. The circumference of the 
membrane is attached to a fixed rim and it is inflated by supplying hydrostatic pressure from below. The external magnetic field is supplied by a fixed dipole placed on the $Y^{3}$ axis as shown in Figure 2a. The deformation is assumed to be symmetric with respect to central $Y^{3}$ axis, hence studying the deformation of one curve connecting the centre to the circumference is sufficient to understand the deformation behaviour of the whole membrane.

The derivations below follows a procedure similar to that in (Reddy and Saxena, 2017, 2018).

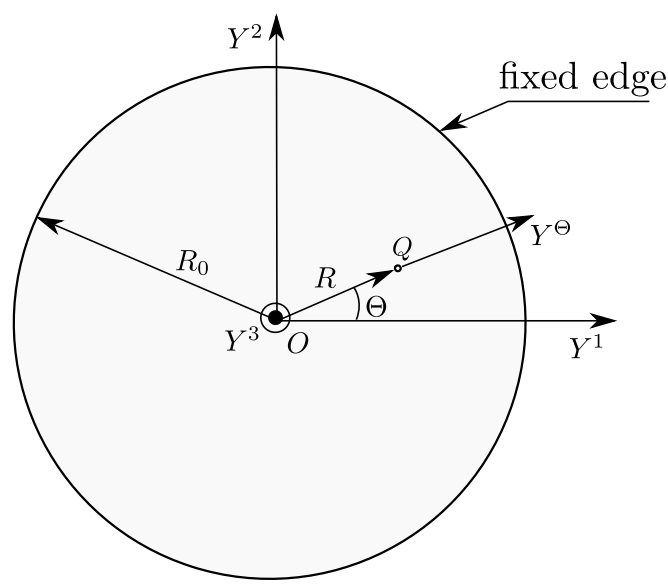

reference configuration

(top view)

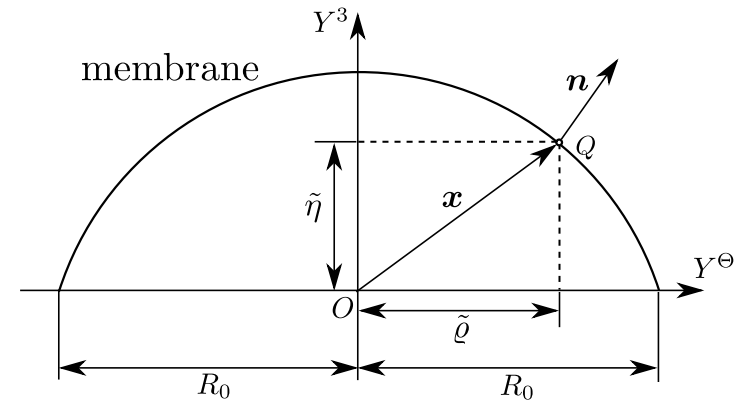

deformed configuration (side view)

Figure 1: Circular membrane (a) before and (b) after deformation. Radius of the undeformed membrane clamped at its edge is $R_{0}$.

\subsection{Reference Configuration}

The position vector of a point in the flesh of the undeformed circular membrane $\boldsymbol{X}$ is

$$
\boldsymbol{X}=R \cos \Theta \boldsymbol{E}_{1}+R \sin \Theta \boldsymbol{E}_{2}+\Xi \boldsymbol{E}_{3},
$$

where $\boldsymbol{E}_{i}$ are the orthonormal basis vectors, $R$ is the radial distance of the point and $\Xi$ is the distance of the point from the mid-surface (given by $\Xi=0$ ) of the membrane along $\boldsymbol{E}_{3}$.

Upon writing bases in the curvilinear system $(R, \Theta, \Xi)$ at the point $\boldsymbol{X}$ as follows

$$
\boldsymbol{G}_{i}=\frac{\partial \boldsymbol{X}}{\partial X^{i}}, \quad \text { where }\left(X^{1}, X^{2}, X^{3}\right)=(R, \Theta, \Xi),
$$

we get the nine components $G_{i j}=\boldsymbol{G}_{i} \cdot \boldsymbol{G}_{j}, i, j \in\{1,2,3\}$ of the covariant metric tensor as

$$
G_{i j}=\left[\begin{array}{ccc}
1 & 0 & 0 \\
0 & R^{2} & 0 \\
0 & 0 & 1
\end{array}\right]
$$

\subsection{Deformed Configuration}

Let $\boldsymbol{p}$ denote the position of a point in the flesh of the membrane, $\boldsymbol{x}$ the position of its projection on the mid-surface (along $\boldsymbol{E}_{3}$ ), $\boldsymbol{n}$ be the unit outward normal, with $\tilde{g}_{i j}$ denoting the covariant metric tensor. The variables $\tilde{\varrho}$ and $\tilde{\eta}$ denote the horizontal and vertical distances, respectively, of the point which was originally at $(R, \Theta, \Xi)$. The relation among these quantities can be written as

$$
\boldsymbol{p}=\boldsymbol{x}+\Xi \lambda_{3} \boldsymbol{n},
$$

$$
\text { with } \quad x^{1}=\tilde{\varrho}(R) \cos \Theta, \quad x^{2}=\tilde{\varrho}(R) \sin \Theta, \quad x^{3}=\tilde{\eta}(R) \text {. }
$$




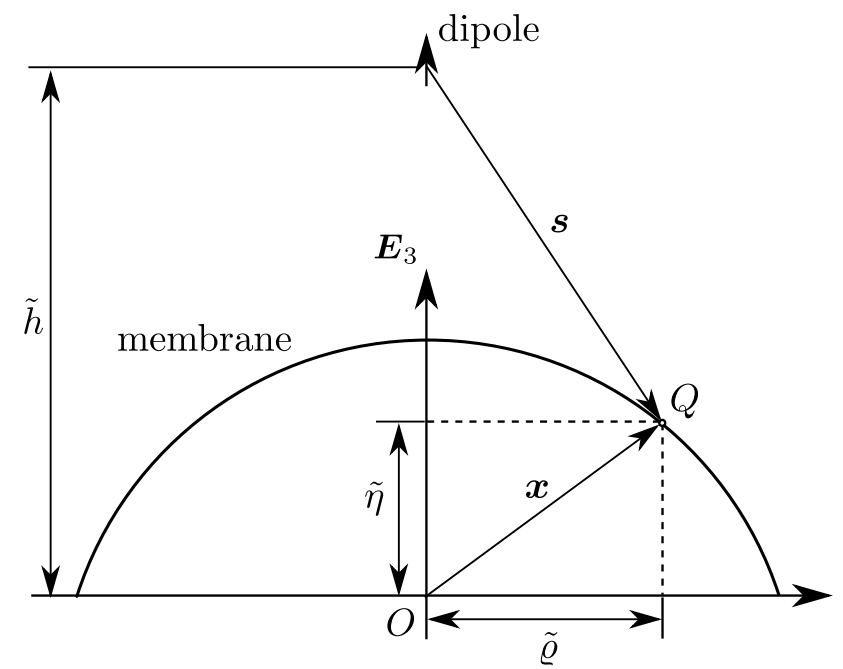

(a)

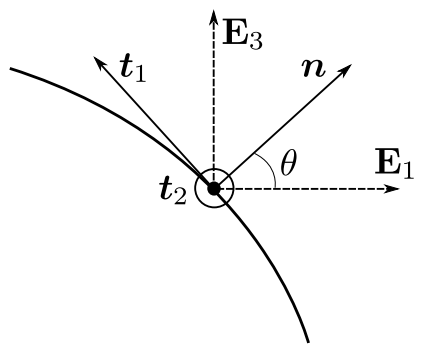

(b)

Figure 2: (a) A deformed configuration of the membrane under the influence of a stationary magnetic dipole. Negative value of $\widetilde{h}$ means the dipole is below the membrane. (b) The local $\boldsymbol{t}_{1}, \boldsymbol{t}_{2}, \boldsymbol{n}$ coordinate system at any point in the membrane is such that $\boldsymbol{t}_{1} \times \boldsymbol{t}_{2}=\boldsymbol{n}$.

where the thickness stretch at the point

$$
\lambda_{3}=\frac{t}{T}
$$

with $t$ and $T$ representing membrane thickness at that point in deformed and reference configurations, respectively. Note that the deformation parameters $\tilde{\varrho}$ and $\tilde{\eta}$ are functions of the radial distance $R$ alone since the azimuthal coordinate of a point doesn't change after deformation and the thickness coordinate $\Xi=0$ on the mid-surface.

Tangent vectors at the points $\boldsymbol{x}$ and $\boldsymbol{p}$ respectively are

$$
\boldsymbol{g}_{i}=\frac{\partial \boldsymbol{x}}{\partial X^{i}} \text { and } \tilde{\boldsymbol{g}}_{i}=\frac{\partial \boldsymbol{p}}{\partial X^{i}}
$$

and the components of the outward normal are

$$
\boldsymbol{n}=\frac{\boldsymbol{g}_{1} \times \boldsymbol{g}_{2}}{\left|\boldsymbol{g}_{1} \times \boldsymbol{g}_{2}\right|}=\frac{1}{\sqrt{g}} \epsilon_{i j k} x_{, 1}^{j} x_{, 2}^{k}
$$

where $\epsilon_{i j k}$ is the permutation symbol and $\sqrt{g}=\tilde{\varrho} \sqrt{\tilde{\varrho}_{, R}^{2}+\tilde{\eta}_{, R}^{2}}$ with $(\cdot)_{, R}=\mathrm{d}(\cdot) / \mathrm{d} R$ everywhere. Expanding the above expression, we get

$$
n_{1}=\frac{1}{\sqrt{g}}\left(-\tilde{\varrho} \tilde{\eta}_{, R} \cos \Theta\right), \quad n_{2}=\frac{1}{\sqrt{g}}\left(-\tilde{\varrho} \tilde{\eta}_{, R} \sin \Theta\right), \quad n_{3}=\frac{1}{\sqrt{g}}\left(\tilde{\varrho} \tilde{\varrho}_{, R}\right) .
$$

Using eqns. (4) and (7), the components $\tilde{g}_{i j}=\tilde{\boldsymbol{g}}_{i} \cdot \tilde{\boldsymbol{g}}_{j}, i, j \in\{1,2,3\}$ of the covariant metric tensor in the deformed state are given as

$$
\tilde{g}_{i j}=\left[\begin{array}{ccc}
\tilde{\varrho}_{, R}^{2}+\tilde{\eta}_{, R}^{2} & 0 & 0 \\
0 & \tilde{\varrho}^{2} & 0 \\
0 & 0 & \lambda_{3}^{2}
\end{array}\right],
$$

where we assume that any length-scale along the thickness coordinate is much smaller than the lengthscales in the plane of membrane. However, the derivatives with respect to the thickness coordinate $\Xi$ are not neglected. Now the corresponding deformation gradient $\mathbf{F}$ and the right Cauchy-Green tensor $\mathbf{C}=\mathbf{F}^{T} \mathbf{F}$ can be written as follows.

$$
\mathbf{F}=\tilde{\boldsymbol{g}}_{i} \otimes \boldsymbol{G}^{i}, \quad \mathbf{C}=\tilde{g}_{i j} \boldsymbol{G}^{i} \otimes \boldsymbol{G}^{j}
$$


Using eqns. (3) and (6), in the Cartesian coordinate system $\left\{\boldsymbol{E}_{1}, \boldsymbol{E}_{2}, \boldsymbol{E}_{3}\right\}$, right Cauchy-Green tensor C can be represented as

$$
\mathbf{C}=\left[\begin{array}{ccc}
\tilde{\varrho}_{, R}^{2}+\tilde{\eta}_{, R}^{2} & 0 & 0 \\
0 & \frac{\tilde{\varrho}^{2}}{R^{2}} & 0 \\
0 & 0 & \lambda_{3}^{2}
\end{array}\right] .
$$

Hence the in-plane principal stretches, square roots of two of the eigenvalues of $\mathbf{C}, \lambda_{1}$ and $\lambda_{2}$ at a point become

$$
\lambda_{1}=\sqrt{\tilde{\varrho}_{, R}^{2}+\tilde{\eta}_{, R}^{2}}, \quad \lambda_{2}=\frac{\tilde{\varrho}}{R},
$$

while the third eigenvalue is the square of the thickness stretch, $\lambda_{3}$. Introducing the non-dimensional parameters

$$
r=R / R_{0}, \quad \varrho=\tilde{\varrho} / R_{0}, \quad \eta=\tilde{\eta} / R_{0},
$$

the principal stretches become

$$
\lambda_{1}=\sqrt{\varrho^{\prime 2}+\eta^{\prime 2}}, \quad \lambda_{2}=\frac{\varrho}{r}
$$

where $(\cdot)^{\prime}=\mathrm{d}(\cdot) / \mathrm{d} r$ everywhere.

\section{Equations of equilibrium}

\subsection{Total potential energy and its variation}

We take the variational formulation as presented in, for example, (Kankanala and Triantafyllidis, 2004) to study the present case of weakly magnetized isotropic circular membrane. As mentioned earlier, the total free energy is based on the deformation gradient (or equivalently, the principal stretches $\lambda_{1}, \lambda_{2}, \lambda_{3}$ ) and magnetization per unit mass. We refer the reader to the papers by Reddy and Saxena $(2017,2018)$ and Barham et al. (2007, 2008) for detailed derivations.

The total potential energy $(E)$ of the membrane under consideration can be written as follows.

$$
E=T \int_{\Omega} \rho \psi \mathrm{d} A-T \mu_{0} \int_{\Omega} \mathbf{m} \cdot \mathbf{h}_{a} \mathrm{~d} A-\int_{V_{0}}^{V_{0}+\Delta V} \tilde{P} \mathrm{~d} V
$$

where $\rho$ is the mass density, $\psi(\mathbf{F}, \boldsymbol{\mu})$ the free energy per unit mass defined in the formulation based on magnetization, $T$ the thickness of the undeformed membrane, $\boldsymbol{\mu}$ the material magnetization per unit mass, $\mathbf{m}=\rho \boldsymbol{\mu}$ the magnetization per unit current volume, $\mu_{0}$ the permeability of free space, $\mathbf{h}_{a}$ the applied external magnetic field, and $\tilde{P}$ is the pressure. $\Omega$ denotes the surface of the undeformed membrane, $V_{0}$ the enclosed initial volume and $\Delta V$ the change in this enclosed volume.

Using the following relations for a weakly magnetized membrane (self-generated magnetic field is negligible) (Barham et al., 2007),

$$
\frac{\partial \psi}{\partial \boldsymbol{\mu}}=\mu_{0} \mathbf{h}_{a}, \quad \rho \psi(\mathbf{F}, \boldsymbol{\mu}) \approx \bar{W}+\frac{1}{2} C|\boldsymbol{\mu}|^{2}, \quad C=\frac{\mu_{0} \rho^{2}}{\chi}, \quad \mathbf{m}=\chi \mathbf{h}_{a},
$$

the total energy may be rewritten as

$$
E[\varrho, \eta]=\int_{0}^{2 \pi} \int_{0}^{R_{0}} \bar{W} T R \mathrm{~d} R \mathrm{~d} \Theta-\frac{\chi}{2} \int_{0}^{2 \pi} \int_{0}^{R_{0}} \mu_{0}\left|\mathbf{h}_{a}\right|^{2} T R \mathrm{~d} R \mathrm{~d} \Theta-\int_{V_{0}}^{V_{0}+\Delta V} \tilde{P} \mathrm{~d} V,
$$

where $\bar{W}$ is the strain energy per unit undeformed volume and $\chi$ is the magnetic susceptibility of the material per unit undeformed volume.

Let the first term, elastic strain energy be denoted by $E_{e}$, the second term, magnetic field energy by $E_{m}$, and the third term, pressure work by $E_{p}$. Note that the strain and magnetic field energies 
are calculated over the reference configuration while the pressure work is computed over the current configuration.

Let the strain energy density function be expressed in terms of the principal stretches as follows

$$
\bar{W}\left(\lambda_{1}, \lambda_{2}, \lambda_{3}\right)=\widetilde{W}\left(\tilde{\varrho}, \frac{\mathrm{d} \varrho}{\mathrm{d} R}, \tilde{\eta}, \frac{\mathrm{d} \tilde{\eta}}{\mathrm{d} R}\right)=W\left(\varrho, \varrho^{\prime}, \eta, \eta^{\prime}\right)
$$

Then using the boundary conditions, due to the geometry and the rotational symmetry about the axis $Y^{3}$,

$$
\varrho=1, \eta=0, \quad \text { at } \quad r=1, \quad \varrho=0, \eta^{\prime}=0, \quad \text { at } \quad r=0,
$$

we can take the first variation of $E_{e}$ to arrive at

$$
\delta E_{e}=\int_{0}^{2 \pi} \int_{0}^{1}\left[\left[-\frac{\mathrm{d}}{\mathrm{d} r}\left(r \frac{\partial W}{\partial \varrho^{\prime}}\right)+r \frac{\partial W}{\partial \varrho}\right] \delta \varrho+\left[-\frac{\mathrm{d}}{\mathrm{d} r}\left(r \frac{\partial W}{\partial \eta^{\prime}}\right)+r \frac{\partial W}{\partial \eta}\right] \delta \eta\right] T R_{0}^{2} \mathrm{~d} r \mathrm{~d} \Theta
$$

A variation in the potential energy of the inflating gas with net internal pressure $\tilde{P}$ can be written as (Steigmann, 1990, Tielking, 1975)

$$
\delta E_{p}=-\int_{0}^{2 \pi} \int_{0}^{R_{0}}[\tilde{P} \boldsymbol{n} \mathrm{d} a] \cdot \delta \boldsymbol{x}
$$

where $\mathrm{d} a=\sqrt{g} \mathrm{~d} R \mathrm{~d} \Theta$ is the area of a differential element on the deformed mid-surface $(\xi=0)$ with unit normal $\boldsymbol{n}$. Using the eqns. (4b) and (7), we get

$$
\delta E_{p}=\int_{0}^{2 \pi} \int_{0}^{1} \tilde{P} R_{0}^{3}\left[\varrho \eta^{\prime} \delta \varrho-\varrho \varrho^{\prime} \delta \eta\right] \mathrm{d} r \mathrm{~d} \Theta
$$

\subsubsection{Energy due to magnetic field of dipole}

A non-uniform magnetic field is generated in the space by placing a magnetic dipole above or below the circular membrane as shown in Figure 2a. A negative numerical value of the height of the dipole, $\tilde{h}$ means the dipole is below the membrane.

Magnetic field intensity due to the dipole $D \boldsymbol{E}_{3}$ of strength $D$ at a point $\boldsymbol{x}$ is

$$
\boldsymbol{h}_{a}(\boldsymbol{x})=D\left[\frac{3\left[\boldsymbol{s} \cdot \boldsymbol{E}_{3}\right] \boldsymbol{s}}{s^{5}}-\frac{\boldsymbol{E}_{3}}{s^{3}}\right],
$$

where $s$ is the position vector of the point relative to the dipole and $s$, its magnitude. Based on the configuration described in Figure 2b, the position vector $s$ can be written in the local coordinate system as

$$
\boldsymbol{s}=[\widetilde{\varrho} \cos \theta-[\widetilde{h}-\widetilde{\eta}] \sin \theta] \boldsymbol{n}-[\widetilde{\varrho} \sin \theta+[\widetilde{h}-\widetilde{\eta}] \cos \theta] \boldsymbol{t}_{1}
$$

The magnetic field intensity can be written in terms of the local coordinate system as

$$
\begin{aligned}
\boldsymbol{h}_{a} & =\frac{3 D[\widetilde{\eta}-\widetilde{h}]}{s^{5}} \boldsymbol{s}-\frac{D}{s^{3}}\left[\boldsymbol{n} \sin \theta+\boldsymbol{t}_{1} \cos \theta\right], \\
& =\frac{D}{s^{3}}\left[\left[\frac{3}{s^{2}}[\widetilde{\eta}-\widetilde{h}][\widetilde{\varrho} \cos \theta-[\widetilde{h}-\widetilde{\eta}] \sin \theta]-\sin \theta\right] \boldsymbol{n}\right. \\
& \left.-\left[\frac{3}{s^{2}}[\widetilde{\eta}-\widetilde{h}][\widetilde{\varrho} \sin \theta+[\widetilde{h}-\widetilde{\eta}] \cos \theta]+\cos \theta\right] \boldsymbol{t}_{1}\right] .
\end{aligned}
$$


Since $s^{2}=\tilde{\varrho}^{2}+[\tilde{h}-\tilde{\eta}]^{2}$, square of the magnitude of the magnetic field intensity can be written as

$$
\left|\boldsymbol{h}_{a}\right|^{2}=D^{2} \frac{\tilde{\varrho}^{2}+4[\tilde{\eta}-\tilde{h}]^{2}}{\left[\tilde{\varrho}^{2}+[\tilde{\eta}-\tilde{h}]^{2}\right]^{4}}
$$

or using the non-dimensional parameters defined in eqn. (13) and defining $h=\frac{\tilde{h}}{R_{0}}$,

$$
\left|\boldsymbol{h}_{a}\right|^{2}=\frac{D^{2}}{R_{0}^{6}}\left[\frac{\varrho^{2}+4[\eta-h]^{2}}{\left[\varrho^{2}+[\eta-h]^{2}\right]^{4}}\right] .
$$

Hence the magnetic energy associated with the magnetic field due to the dipole is given as

$$
E_{m}=-\frac{\chi}{2} \int_{0}^{2 \pi} \int_{0}^{1} \mathcal{H} T R_{0}^{2} r \mathrm{~d} r \mathrm{~d} \Theta
$$

where $\mathcal{H}$ is defined as

$$
\mathcal{H}=\frac{\mu_{0} D^{2}}{R_{0}^{6}}\left[\frac{\varrho^{2}+4[\eta-h]^{2}}{\left[\varrho^{2}+[\eta-h]^{2}\right]^{4}}\right] .
$$

A variation in the magnetic energy can therefore be written as

$$
\delta E_{m}=-\frac{\chi}{2} \int_{0}^{2 \pi} \int_{0}^{1}\left[\frac{\partial \mathcal{H}}{\partial \varrho} \delta \varrho+\frac{\partial \mathcal{H}}{\partial \eta} \delta \eta\right] T R_{0}^{2} r \mathrm{~d} r \mathrm{~d} \Theta
$$

where

$$
\frac{\partial \mathcal{H}}{\partial \varrho}=\frac{-6 \mu_{0} D^{2}}{R_{0}^{6}} \frac{\varrho\left[\varrho^{2}+5[\eta-h]^{2}\right]}{\left[\varrho^{2}+[\eta-h]^{2}\right]^{5}}, \quad \frac{\partial \mathcal{H}}{\partial \eta}=\frac{-24 \mu_{0} D^{2}}{R_{0}^{6}} \frac{[\eta-h]^{3}}{\left[\varrho^{2}+[\eta-h]^{2}\right]^{5}} .
$$

\subsubsection{Constitutive model for the elastic strain energy}

We study the deformation of the circular membrane using the three-term Ogden model for the elastic strain energy density $\bar{W}$ in eqn. (16) $)_{2}$ and further assume the material to be incompressible $\left(\lambda_{1} \lambda_{2} \lambda_{3}=1\right)$. Thus we have

$$
\widehat{W}\left(\lambda_{1}, \lambda_{2}\right)=\bar{W}\left(\lambda_{1}, \lambda_{2}, \frac{1}{\lambda_{1} \lambda_{2}}\right) .
$$

The strain energy density proposed by Ogden (1972) to model nonlinear elastic solids is given by

$$
\widehat{W}=\sum_{k=1}^{K} \frac{\mu_{k}}{\alpha_{k}}\left[\lambda_{1}^{\alpha_{k}}+\lambda_{2}^{\alpha_{k}}+\left[\frac{1}{\lambda_{1} \lambda_{2}}\right]^{\alpha_{k}}-3\right],
$$

with the conditions $\sum_{k} \mu_{k} \alpha_{k}=2 \mu$ and $\mu_{k} \alpha_{k}>0$. Choosing $K=3$, we define the following nondimensional parameters

$$
\mu_{1}^{*}=\mu_{1} / \mu, \quad \mu_{2}^{*}=\mu_{2} / \mu, \quad \mu_{3}^{*}=\mu_{3} / \mu,
$$

maintaining $\sum_{k} \mu_{k} \alpha_{k}=2 \mu$.

\subsection{Governing equations}

The system attains equilibrium when the total potential energy is at a local extremum, that is

$$
\delta E=\delta E_{e}+\delta E_{m}+\delta E_{p}=0 .
$$


Upon separating the coefficients of $\delta \varrho$ and $\delta \eta$ from equations (20), (22) and (30), we arrive at the following governing equations

$$
\begin{gathered}
\frac{\mathrm{d}}{\mathrm{d} r}\left(r \frac{\partial W}{\partial \varrho^{\prime}}\right)-r \frac{\partial W}{\partial \varrho}+\frac{\chi}{2} \frac{\partial \mathcal{H}}{\partial \varrho} r-\frac{R_{0}}{T} \tilde{P} \varrho \eta^{\prime}=0, \\
\frac{\mathrm{d}}{\mathrm{d} r}\left(r \frac{\partial W}{\partial \eta^{\prime}}\right)-r \frac{\partial W}{\partial \eta}+\frac{\chi}{2} \frac{\partial \mathcal{H}}{\partial \eta} r+\frac{R_{0}}{T} \tilde{P} \varrho \varrho^{\prime}=0 .
\end{gathered}
$$

Defining a non-dimensional magnetic energy parameter in terms of the shear modulus $\mu$ as

$$
\mathcal{M}=\frac{\mu_{0} D^{2}}{R_{0}^{6} \mu},
$$

and a non-dimensional pressure as

$$
P=\frac{\tilde{P} R_{0}}{\mu T},
$$

using the relations (31), the governing equations (36) attain the following form.

$$
\begin{gathered}
\frac{1}{\mu} \frac{\mathrm{d}}{\mathrm{d} r}\left(\frac{\varrho^{\prime} r}{\lambda_{1}} \frac{\partial \widehat{W}}{\partial \lambda_{1}}\right)-\frac{1}{\mu} \frac{\partial \widehat{W}}{\partial \lambda_{2}}-3 \chi \mathcal{M} r \frac{\varrho\left[\varrho^{2}+5[\eta-h]^{2}\right]}{\left[\varrho^{2}+[\eta-h]^{2}\right]^{5}}-P \varrho \eta^{\prime}=0, \\
\frac{1}{\mu} \frac{\mathrm{d}}{\mathrm{d} r}\left(\frac{\eta^{\prime} r}{\lambda_{1}} \frac{\partial \widehat{W}}{\partial \lambda_{1}}\right)-12 \chi \mathcal{M} r \frac{[\eta-h]^{3}}{\left[\varrho^{2}+[\eta-h]^{2}\right]^{5}}+P \varrho \varrho^{\prime}=0 .
\end{gathered}
$$

\subsection{Stability of equilibrium}

A necessary condition for the equilibrium state obtained from solution of the governing equations (39) to be a minimizer of the energy functional (17) is that the second variation be positive definite that results in the necessary condition that the matrix

$$
\mathbf{P}=\frac{1}{2}\left[\begin{array}{cc}
\mathcal{F}_{\varrho^{\prime}} \varrho^{\prime} & \mathcal{F}_{\varrho^{\prime} \eta^{\prime}} \\
\mathcal{F}_{\eta^{\prime} \varrho^{\prime}} & \mathcal{F}_{\eta^{\prime} \eta^{\prime}}
\end{array}\right]
$$

with

$$
\mathcal{F}=\widehat{W} T R_{0}^{2} r-\frac{\chi}{2} \mu_{0}\left|\mathbf{h}_{a}\right|^{2} T R_{0}^{2} r+\frac{1}{3} \tilde{P} R_{0}^{3}\left[\varrho^{2} \eta^{\prime}-\varrho \varrho^{\prime} \eta\right]
$$

being the integrand in equation (17) be positive definite for all $r \in[0,1]$. A sufficient condition for minimization (Gelfand and Fomin, 2000, Ch. 5) is that a non-zero solution to the following differential equation exists and is invertible for all $r \in[0,1]$

$$
-\frac{\mathrm{d}}{\mathrm{d} r}\left(\mathbf{P} \mathbf{U}^{\prime}\right)+\mathbf{Q U}=0, \quad \mathbf{U}(0)=\left[\begin{array}{ll}
0 & 0 \\
0 & 0
\end{array}\right], \quad \mathbf{U}^{\prime}(0)=\left[\begin{array}{ll}
1 & 0 \\
0 & 1
\end{array}\right],
$$

where

$$
\mathrm{Q}=\frac{1}{2}\left[\begin{array}{cc}
\mathcal{F}_{\varrho \varrho} & \mathcal{F}_{\varrho \eta} \\
\mathcal{F}_{\eta \varrho} & \mathcal{F}_{\eta \eta}
\end{array}\right]-\frac{1}{2} \frac{\mathrm{d}}{\mathrm{d} r}\left(\left[\begin{array}{cc}
\mathcal{F}_{\varrho \varrho^{\prime}} & \mathcal{F}_{\varrho \eta^{\prime}} \\
\mathcal{F}_{\eta \varrho^{\prime}} & \mathcal{F}_{\eta \eta^{\prime}}
\end{array}\right]\right) .
$$

Upon defining

$$
\begin{gathered}
\mathbf{P}=\frac{1}{2}\left[\begin{array}{ll}
P_{1} & P_{2} \\
P_{3} & P_{4}
\end{array}\right], \quad \mathbf{Q}=\frac{1}{2}\left[\begin{array}{ll}
Q_{1} & Q_{2} \\
Q_{3} & Q_{4}
\end{array}\right], \quad \mathbf{U}=\left[\begin{array}{ll}
U_{1} & U_{2} \\
U_{3} & U_{4}
\end{array}\right], \\
\left\{U_{1}, U_{1}^{\prime}, U_{2}, U_{2}^{\prime}, U_{3}, U_{3}^{\prime}, U_{4}, U_{4}^{\prime}\right\}=\left\{u_{1}, u_{2}, u_{3}, u_{4}, u_{5}, u_{6}, u_{7}, u_{8}\right\},
\end{gathered}
$$


the differential equation (42) can be written as

$$
\left[\begin{array}{cccccccc}
1 & 0 & 0 & 0 & 0 & 0 & 0 & 0 \\
0 & -P_{1} & 0 & 0 & 0 & -P_{2} & 0 & 0 \\
0 & 0 & 1 & 0 & 0 & 0 & 0 & 0 \\
0 & 0 & 0 & -P_{1} & 0 & 0 & 0 & -P_{2} \\
0 & 0 & 0 & 0 & 1 & 0 & 0 & 0 \\
0 & -P_{3} & 0 & 0 & 0 & -P_{4} & 0 & 0 \\
0 & 0 & 0 & 0 & 0 & 0 & 1 & 0 \\
0 & 0 & 0 & -P_{3} & 0 & 0 & 0 & -P_{4}
\end{array}\right]\left[\begin{array}{c}
u_{1}^{\prime} \\
u_{2}^{\prime} \\
u_{3}^{\prime} \\
u_{4}^{\prime} \\
u_{5}^{\prime} \\
u_{6}^{\prime} \\
u_{7}^{\prime} \\
u_{8}^{\prime}
\end{array}\right]=\left[\begin{array}{c}
u_{2} \\
P_{1}^{\prime} u_{2}+P_{2}^{\prime} u_{6}-Q_{1} u_{1}-Q_{2} u_{5} \\
u_{4} \\
P_{1}^{\prime} u_{4}+P_{2}^{\prime} u_{8}-Q_{1} u_{3}-Q_{2} u_{7} \\
u_{6}^{\prime} u_{2}+P_{4}^{\prime} u_{6}-Q_{3} u_{1}-Q_{4} u_{5} \\
u_{8} \\
P_{3}^{\prime} u_{4}+P_{4}^{\prime} u_{8}-Q_{3} u_{3}-Q_{4} u_{7}
\end{array}\right],
$$

Explicit calculations of the entries of matrices $\mathbf{P}$ and $\mathbf{Q}$ for the present case of Ogden energy density function are presented in Appendix A.

\subsection{Magnetoelastic stresses and wrinkling}

The expression for total magnetoelastic (Cauchy) stress tensor is given by (Steigmann, 2004)

$$
\boldsymbol{\sigma}=\rho \psi_{, \mathbf{F}} \mathbf{F}^{T}+\mu_{0}\left[\mathbf{h} \otimes \mathbf{h}-\frac{1}{2}[\mathbf{h} \cdot \mathbf{h}] \boldsymbol{i}\right]+\mu_{0} \mathbf{h} \otimes \mathbf{m}-q \boldsymbol{i},
$$

where $q$ is a Lagrange multiplier due to the constraint of incompressibility. The Maxwell stress due to magnetic field is given as

$$
\boldsymbol{\sigma}_{m}=\mu_{0}\left[\mathbf{h} \otimes \mathbf{h}-\frac{1}{2}[\mathbf{h} \cdot \mathbf{h}] \boldsymbol{i}\right] .
$$

We consider the local coordinate system with two axes in the plane of membrane and the third axis as the outward normal $\boldsymbol{n}$ from equation (7) as shown in Figure 2b. Writing the balance of traction on the inner surface while using equation (16) gives

$$
\left[\boldsymbol{\sigma}-\boldsymbol{\sigma}_{m}\right] \boldsymbol{n}=-\widetilde{P} \boldsymbol{n}, \quad \Rightarrow \bar{W}_{, \mathbf{F}} \mathbf{F}^{T} \boldsymbol{n}+\mu_{0} \chi[\mathbf{h} \cdot \boldsymbol{n}] \mathbf{h}-q \boldsymbol{n}=-\widetilde{P} \boldsymbol{n} .
$$

Taking an inner product of the above equation with the unit vector $\boldsymbol{n}$, we can evaluate the Lagrange multiplier $q$ as

$$
q=\widetilde{P}+\mu_{0} \chi[\mathbf{h} \cdot \boldsymbol{n}]^{2}+\left[\bar{W}_{, \mathbf{F}} \mathbf{F}^{T} \boldsymbol{n}\right] \cdot \boldsymbol{n},
$$

which gives the total stress within the membrane as

$$
\boldsymbol{\sigma}=\bar{W}_{, \mathbf{F}} \mathbf{F}^{T}+\mu_{0}\left[\mathbf{h} \otimes \mathbf{h}-\frac{1}{2}[\mathbf{h} \cdot \mathbf{h}] \boldsymbol{i}\right]+\mu_{0} \mathbf{h} \otimes \mathbf{m}-\left[\widetilde{P}+\mu_{0} \chi[\mathbf{h} \cdot \boldsymbol{n}]^{2}+\left[\bar{W}_{, \mathbf{F}} \mathbf{F}^{T} \boldsymbol{n}\right] \cdot \boldsymbol{n}\right] \boldsymbol{i} .
$$

Within the plane of the membrane (spanned by $\boldsymbol{t}_{1}$ and $\boldsymbol{t}_{2}$ ), the components of total stress are given by

$$
s_{11}=\left[\boldsymbol{\sigma} \boldsymbol{t}_{1}\right] \cdot \boldsymbol{t}_{1}, \quad s_{12}=\left[\boldsymbol{\sigma} \boldsymbol{t}_{2}\right] \cdot \boldsymbol{t}_{1}, \quad s_{21}=\left[\boldsymbol{\sigma} \boldsymbol{t}_{1}\right] \cdot \boldsymbol{t}_{2}, \quad s_{22}=\left[\boldsymbol{\sigma} \boldsymbol{t}_{2}\right] \cdot \boldsymbol{t}_{2} .
$$

Using equation (50), we can write these components explicitly as

$$
s_{11}=\left[\bar{W}_{, \mathbf{F}} \mathbf{F}^{T} \boldsymbol{t}_{1}\right] \cdot \boldsymbol{t}_{1}+\mu_{0}[1+\chi] h_{t}^{2}-\frac{\mu_{0}\left[h_{t}^{2}+h_{n}^{2}\right]}{2}-\left[\widetilde{P}+\mu_{0} \chi h_{n}^{2}+\left[\bar{W}_{, \mathbf{F}} \mathbf{F}^{T} \boldsymbol{n}\right] \cdot \boldsymbol{n}\right]
$$




$$
\begin{aligned}
& =-\widetilde{P}+\lambda_{1} w_{1}-\lambda_{3} w_{3}+\mu_{0}\left[\chi+\frac{1}{2}\right]\left[h_{t}^{2}-h_{n}^{2}\right], \\
s_{12} & =s_{21}=0, \\
s_{22} & =-\widetilde{P}+\lambda_{2} w_{2}-\lambda_{3} w_{3}-\frac{\mu_{0}\left[h_{t}^{2}+h_{n}^{2}\right]}{2}-\mu_{0} \chi h_{n}^{2} .
\end{aligned}
$$

where we have used the notation $\mathbf{h}=h_{n} \boldsymbol{n}+h_{t} \boldsymbol{t}_{1}$ as a shorter version of equation (25), $w_{i}=\partial \bar{W} / \partial \lambda_{i}$ and the diagonal terms $s_{12}=s_{21}=0$ because $\boldsymbol{F}$ is represented by a diagonal matrix in the local $\left(\boldsymbol{n}, \boldsymbol{t}_{1}, \boldsymbol{t}_{2}\right)$ coordinate system.

In order for the membrane to remain taut, both the in-plane principal stress components $s_{11}$ and $s_{22}$ should be positive. Either of them becoming negative gives the indication of wrikling instability in that region.

For the Ogden material described in equation (33) used in this work, the above expressions for principal stresses reduce to

$$
\begin{aligned}
& s_{11}=-\widetilde{P}+\mu_{0}\left[\chi+\frac{1}{2}\right]\left[h_{t}^{2}-h_{n}^{2}\right]+\sum_{k=1}^{3} \mu_{k}\left[\lambda_{1}^{\alpha_{k}}-\frac{1}{\lambda_{1}^{\alpha_{k}} \lambda_{2}^{\alpha_{k}}}\right], \\
& s_{22}=-\widetilde{P}-\frac{\mu_{0}\left[h_{t}^{2}+h_{n}^{2}\right]}{2}-\mu_{0} \chi h_{n}^{2}+\sum_{k=1}^{3} \mu_{k}\left[\lambda_{2}^{\alpha_{k}}-\frac{1}{\lambda_{1}^{\alpha_{k}} \lambda_{2}^{\alpha_{k}}}\right] .
\end{aligned}
$$

We also note that the criteria mentioned here is different from that used by Reddy and Saxena (2018) who only considered the mechanical component of stress to determine the onset of wrinkling. It has been demonstrated (Dorfmann and Ogden, 2014) that the total magnetoelastic stress gives a more accurate description of internal forces due to coupled loading and therefore we use it for our computations in this paper.

\subsection{Numerical solution procedure}

The governing equations (39) need to be solved for the non-dimensional quantities $\varrho, \varrho^{\prime}, \eta$, and $\eta^{\prime}$ for the magnetic and pressure loading parameters $\mathcal{M}$ and $P$, respectively using the boundary conditions (19). Circumferential stretch $\lambda_{2}=\varrho / r$ becomes indeterminate at the pole $(r=0)$. To avoid this, we choose the following set of parameters as proposed in (Patil and Dasgupta, 2013)

$$
u=r \lambda_{2}, \quad v=r \lambda_{2}^{\prime}, w=\eta^{\prime}
$$

such that

$$
\varrho=u, \varrho^{\prime}=\lambda_{2}+v,
$$

and start the integration at a very small value of the radius $r$.

Since the pressure-stretch curve for a membrane in general is not monotonic, to trace it past the limit-point, we treat pressure $P$ as an unknown and initiate the numerical solution procedure with a value of $\left.\lambda_{1}\right|_{r=0}=\left.\lambda_{2}\right|_{r=0}=\varrho^{\prime}(0)=\lambda_{0}$, radial stretch at the center of the membrane as an input. Now the coupled second order ordinary differential equations (39) can be converted in to the following system of equations.

$$
\mathbf{A X}^{\prime}=\mathbf{E}
$$

where

$$
\begin{gathered}
\mathbf{X}=(u, v, \eta, w)^{T}, \quad \mathbf{X}^{\prime}=\left(u^{\prime}, v^{\prime}, \eta^{\prime}, w^{\prime}\right)^{T}, \quad(\cdot)^{T} \text { representing transpose } \\
\mathbf{A}=\left(\begin{array}{cccc}
1 & 0 & 0 & 0 \\
0 & \mathcal{A} & 0 & \mathcal{B} \\
0 & 0 & 1 & 0 \\
0 & \mathcal{C} & 0 & \mathcal{D}
\end{array}\right), \mathbf{E}=\left(\begin{array}{c}
\lambda_{2}+v \\
\mathcal{E} \\
w \\
\mathcal{F}
\end{array}\right)
\end{gathered}
$$




$$
\begin{aligned}
& \mathcal{A}=\frac{r}{\lambda_{1}^{2}}\left[\lambda_{1}-\frac{1}{\lambda_{1}}\left[\lambda_{2}+v\right]^{2}\right] \frac{1}{\mu} \frac{\partial \widehat{W}}{\partial \lambda_{1}}+\frac{\lambda_{2}+v}{\lambda_{1}} W_{11}, \\
& \mathcal{B}=-\frac{r w}{\lambda_{1}^{3}}\left[\lambda_{2}+v\right] \frac{1}{\mu} \frac{\partial \widehat{W}}{\partial \lambda_{1}}+\frac{\lambda_{2}+v}{\lambda_{1}} W_{12}, \\
& \mathcal{C}=-\frac{r w}{\lambda_{1}^{3}}\left[\lambda_{2}+v\right] \frac{1}{\mu} \frac{\partial \widehat{W}}{\partial \lambda_{1}}+\frac{w}{\lambda_{1}} W_{11}, \quad \mathcal{D}=\frac{r}{\lambda_{1}^{2}}\left[\lambda_{1}-\frac{w^{2}}{\lambda_{1}}\right] \frac{1}{\mu} \frac{\partial \widehat{W}}{\partial \lambda_{1}}+\frac{w}{\lambda_{1}} W_{12}, \\
& \mathcal{E}=-\mathcal{E}_{1}+\frac{1}{\mu} \frac{\partial \widehat{W}}{\partial \lambda_{2}}+3 \chi \mathcal{M} r \frac{\varrho\left[\varrho^{2}+5[\eta-h]^{2}\right]}{\left[\varrho^{2}+[\eta-h]^{2}\right]^{5}}+P u w, \\
& \mathcal{F}=-\mathcal{F}_{1}+12 \chi \mathcal{M} r \frac{[\eta-h]^{3}}{\left[\varrho^{2}+[\eta-h]^{2}\right]^{5}}-P u\left[\lambda_{2}+v\right], \\
& \mathcal{E}_{1}=\frac{\lambda_{2}+v}{\lambda_{1}}\left[\frac{1}{\mu} \frac{\partial \widehat{W}}{\partial \lambda_{1}}+W_{13}\right]+\frac{1}{\mu} \frac{\partial \widehat{W}}{\partial \lambda_{1}} \frac{v}{\lambda_{1}^{2}}\left[\lambda_{1}-\frac{1}{\lambda_{1}}\left[\lambda_{2}+v\right]^{2}\right] \text {, } \\
& \mathcal{F}_{1}=\frac{w}{\lambda_{1}}\left[\frac{1}{\mu} \frac{\partial \widehat{W}}{\partial \lambda_{1}}+W_{13}\right]-\frac{1}{\mu} \frac{\partial \widehat{W}}{\partial \lambda_{1}} \frac{w}{\lambda_{1}^{3}}\left[\lambda_{2}+v\right] v, \\
& W_{11}=\sum_{k=1}^{3} d_{k}\left(\lambda_{2}+v\right) r, \quad W_{12}=\sum_{k=1}^{3} e_{k}, \\
& W_{13}=\sum_{k=1}^{3}\left[d_{k}\left[\lambda_{2}+v\right] v+\left[\frac{b_{k}}{\lambda_{1} \lambda_{2}^{2}}+c_{k}\right] v\right] \text {, } \\
& d_{k}=\frac{a_{k}}{\lambda_{1}}+\frac{b_{k}}{\lambda_{2} \lambda_{1}^{3}}, \quad e_{k}=a_{k} \frac{r w}{\lambda_{1}}+b_{k} \frac{r w}{\lambda_{2} \lambda_{1}^{3}} \\
& a_{k}=\mu_{k}^{*}\left[\alpha_{k}-1\right] \lambda_{1}^{\alpha_{k}-2}, \quad b_{k}=\frac{\mu_{k}^{*} \alpha_{k}}{\lambda_{2}} \lambda_{3}^{\alpha_{k}-1}, \quad c_{k}=\mu_{k}^{*} \frac{\lambda_{3}^{\alpha_{k}}}{\lambda_{2}^{2}}, \\
& \frac{1}{\mu} \frac{\partial \widehat{W}}{\partial \lambda_{i}}=\sum_{k=1}^{3} \mu_{k}^{*}\left[\lambda_{i}^{\alpha_{k}-1}-\left[\frac{1}{\lambda_{1} \lambda_{2}}\right]^{\alpha_{k}} \frac{1}{\lambda_{i}}\right]
\end{aligned}
$$

with the boundary conditions

$$
u\left(r_{0}\right)=r_{0} \lambda_{0}, \quad u(1)=1, v\left(r_{0}\right)=0, w\left(r_{0}\right)=0,
$$

where $r_{0}=1 \times 10^{-5}$.

The above boundary value problem is converted into an initial value problem using shooting method and the resultant is solved using a fourth-order Runge-Kutta method (via ode45 in MATLAB ${ }^{\circledR}$ ). For a given value of the position of centre $\eta(0)$, we start with a guess pair $\left(\varrho^{\prime}(0), P\right)$, and apply the shooting method to obtain the pair $(\varrho(1), \eta(1))$ which should ideally be $(1,0)$. Note that the value $\varrho^{\prime}(0)>0$ since it represents a stretch and $\varrho^{\prime}(0)>1$ when the membrane is in extension at its center. The correct pair $\left(\varrho^{\prime}(0), P\right)$ that makes the quantity $\sqrt{[\varrho(1)-1]^{2}+\eta(1)^{2}}$ sufficiently close to 0 (within $\left.\mathcal{O}\left(10^{-13}\right)\right)$ is obtained using Nelder-Meads optimization technique (via fminsearch in MATLAB ${ }^{\circledR}$ ). For this correct pair, the Runge-Kutta method gives the values of $\left(\varrho, r \lambda_{2}^{\prime}, \eta, \eta^{\prime}\right)$ over the domain $r \in\left[r_{0}, 1\right]$.

\section{Numerical results}

\subsection{Validating the formulation}

We start with comparing the numerical results obtained through our formulation for purely elastic case $(\mathcal{M}=0)$ with those obtained for the same problem by Verron and Marckmann (2003). As seen in 
Table 1: The dimensionless parameters used in the computations are listed below. Material parameters for the energy density functions are taken from (Ogden, 1972).

\begin{tabular}{cccccccc}
\hline$R_{0} / T$ & $\mu_{1}^{*}$ & $\mu_{2}^{*}$ & $\mu_{3}^{*}$ & $\alpha_{1}$ & $\alpha_{2}$ & $\alpha_{3}$ & $\chi$ \\
\hline 1000 & 1.4910 & 0.0029 & -0.0236 & 1.3 & 5.0 & -2.0 & 2.5 \\
\hline
\end{tabular}

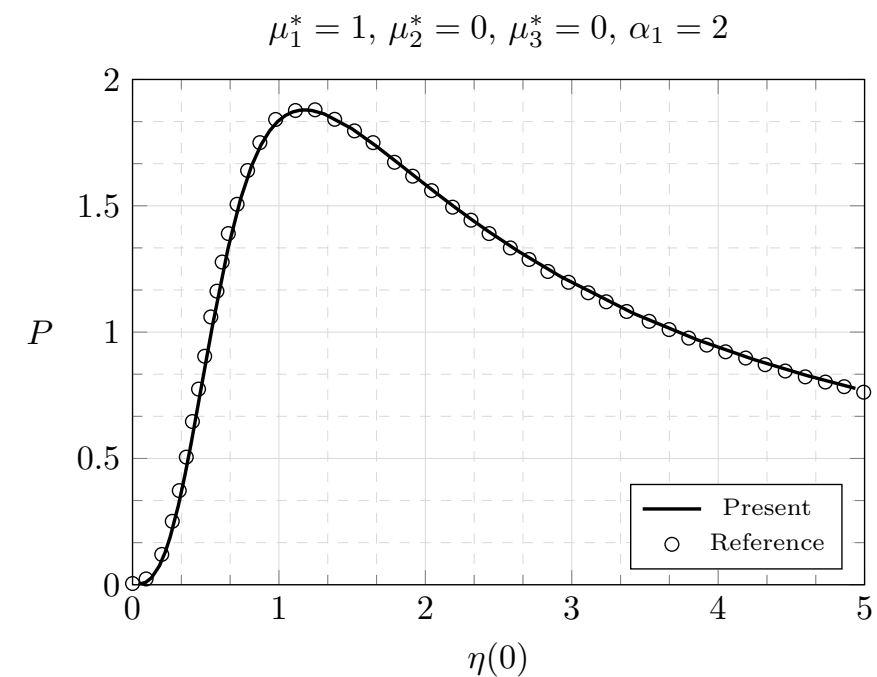

(a) Normalised pressure vs deformation of midpoint.

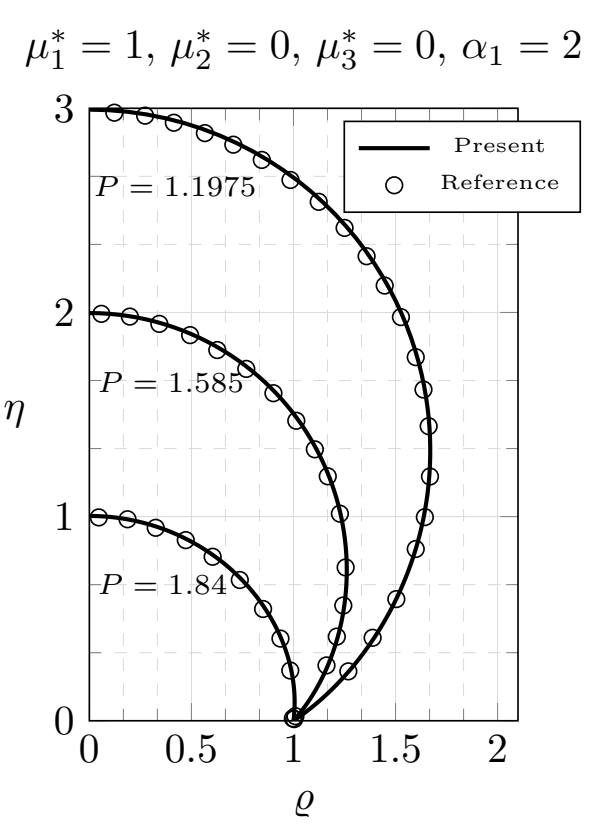

(b) Membrane profiles

Figure 3: Comparison of our numerical solutions with Verron and Marckmann (2003) for purely mechanical deformation using neo-Hookean energy model. Figure (b) includes membrane profiles from post elastic limit-point behaviour.

Figures $3 \mathrm{a}$ and $3 \mathrm{~b}$, the pressure-stretch plot for the centre and the membrane profiles match perfectly with those presented in this reference for a neo-Hookean material (upon substituting $\mu_{2}=\mu_{3}=0$ in Equation (33)) in the post-limit point regime.

\subsection{Inflation under the field of a dipole}

We now solve the complete set of equations (39) using the scheme described in Section 3.5. It is observed in our numerical calculations that the solution converges for all values of $R_{0} / T>100$, thus we choose a conservative value of $R_{0} / T=1000$ in all the computations. Values of the material parameters $\mu_{i}^{*}, \alpha_{i}$ and $\chi$ listed in Table 1 are the same as those used by Ogden (1972), Barham et al. (2008), and Reddy and Saxena (2018).

\subsubsection{Pressure-volume characteristics and limit points}

The variation of pressure required to inflate the membrane with the volume of gas pumped in the presence of a magnetic dipole is shown in Figures 4 and 5 . The purely mechanical case $(\mathcal{M}=0)$ represented by dashed curves in Figure 4 shows monotonic increase of pressure until a maxima (referred to as limit point in the literature) is reached. Beyond this limit point, pumping more gas in the membrane (increase in volume $V$ ) results in a decrease of required pressure until a minima is reached and the pressure rises again with volume for large values of volume.

When the dipole is placed on top of the membrane $(h>0)$, variation of pressure is pretty similar to the purely mechanical case for small volume. Upon reaching large values of volume when the top of the membrane reaches close to the dipole, a sudden drop of pressure is observed resulting in a new limit point. The equilibria obtained beyond this second limit point are most likely unstable as the membrane 
(a)

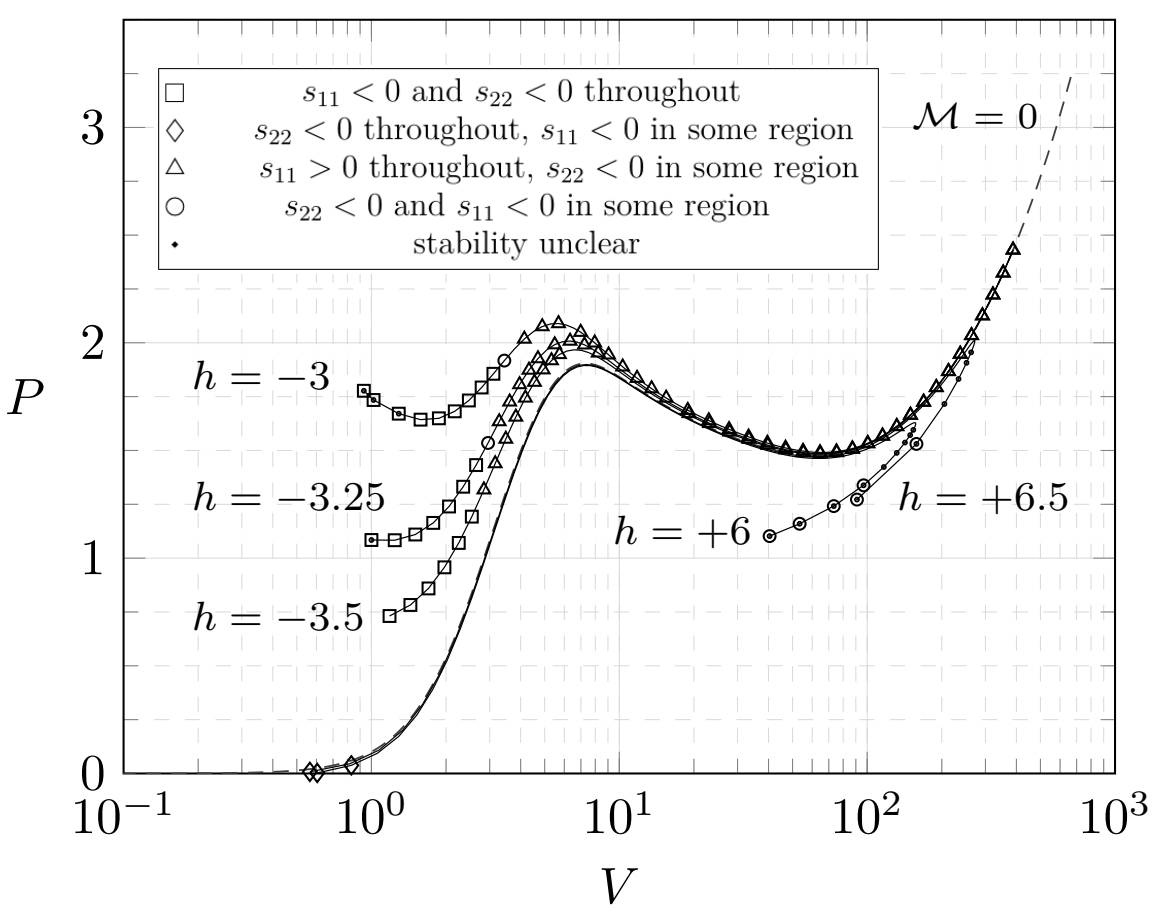

(b)

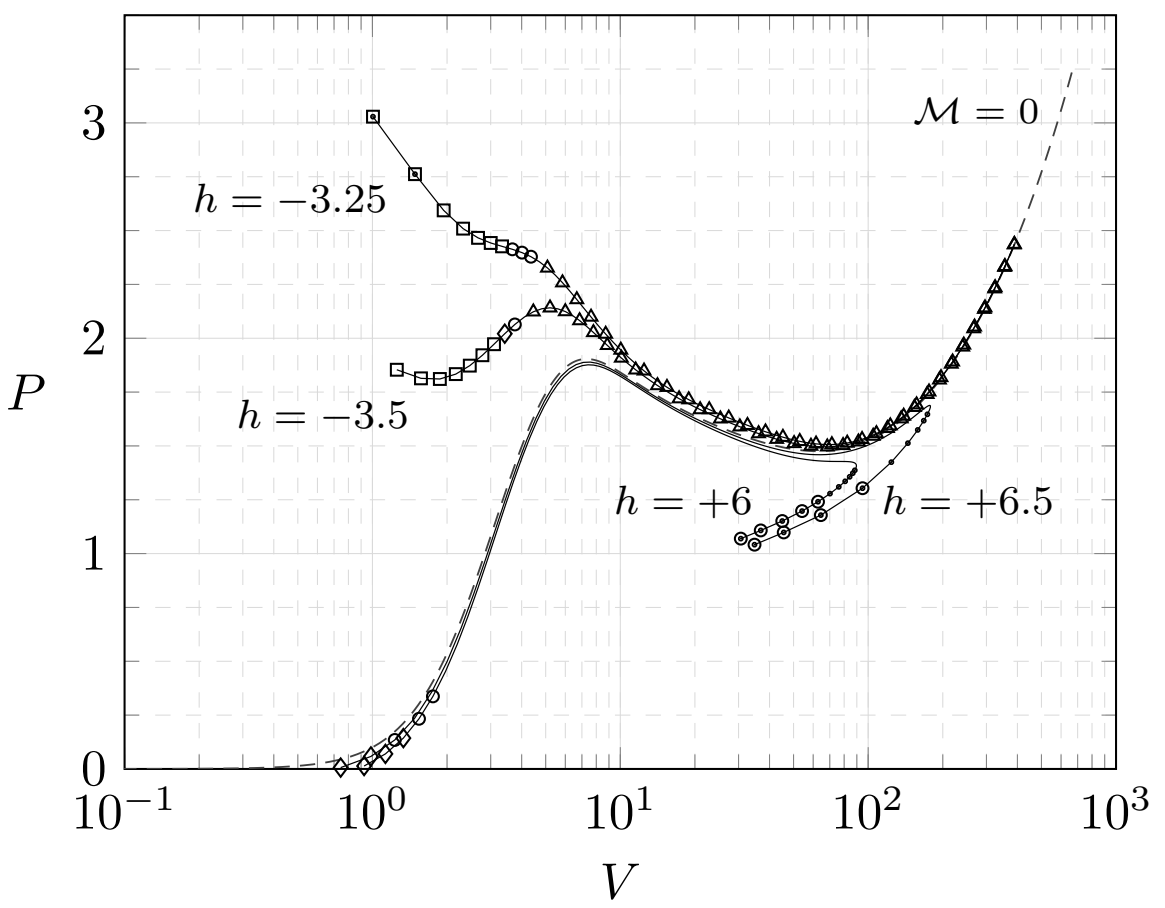

Figure 4: Pressure vs volume curves for two different strengths of dipole above and below the membrane at equal distance (a) $\mathcal{M}=200$, (b) $\mathcal{M}=600$. Dashed curves in both the figures correspond to the purely mechanical case with no dipole. 


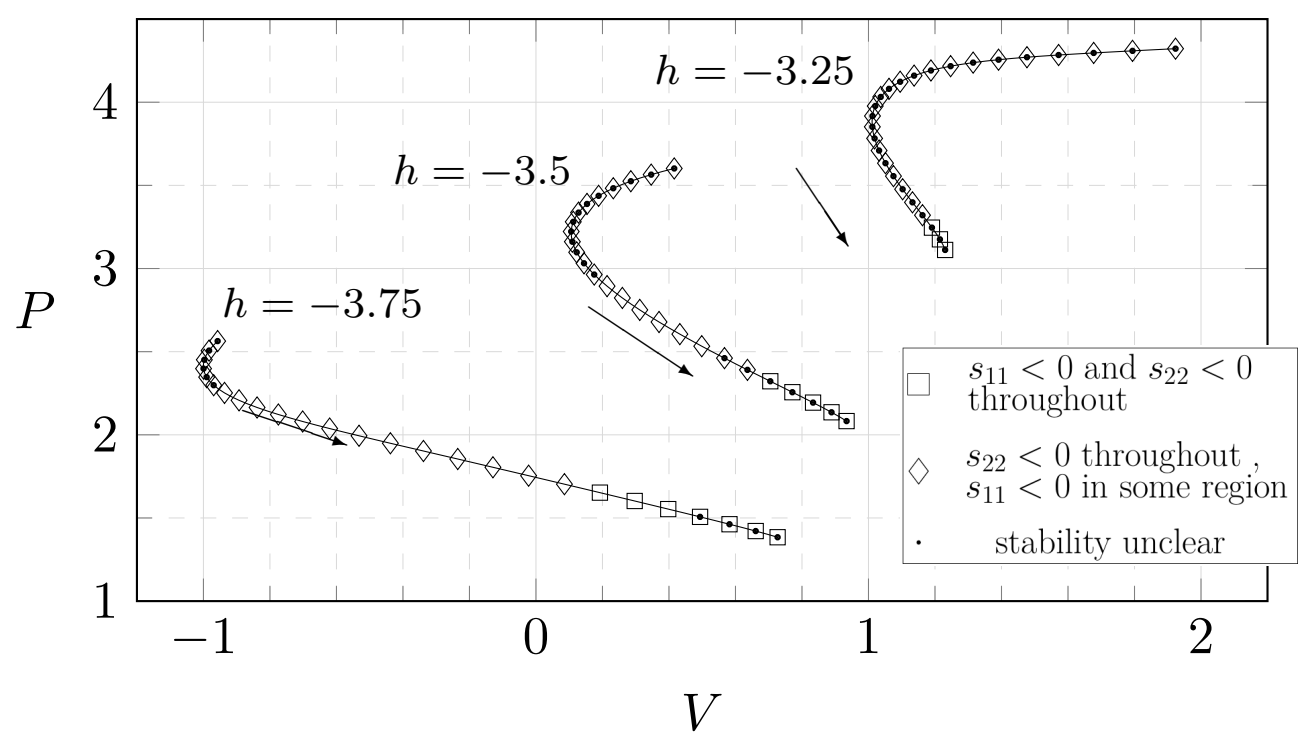

Figure 5: Pressure vs volume curves for small deformations when a strong dipole $\mathcal{M}=600$ is placed below the membrane. Negative values of volume correspond to the cases in which membrane is pulled downwards. Arrows represent the direction of increasing $\eta(0)$ value.

will be dynamically pulled towards the dipole resulting in tremendous increase in magnetic part $E_{m}$ of the total energy as presented in equation (28). Instability of this equilibria is discussed at the end of this section.

The pressure-volume characteristics change significantly when the dipole is placed below the membrane. Curves for small values of inflation in the presence of a strong dipole $\mathcal{M}=600$ are presented in Figure 5. Since membrane can be pulled below towards the dipole $(\eta(0)<0)$, we encounter negative values of volume in these graphs. As the centre of membrane moves up (increase in $\eta(0)$ ), the volume first decreases and then increases while the pressure continues to fall. This additional limit point at the very early stage of inflation is similar to that observed in a similar problem but different geometry (Reddy and Saxena, 2018, Fig. 5c). Continuation of these curves is presented in Figure 4b. For stronger dipole $(\mathcal{M}=600)$ closer to the membrane $(h=-3.25)$ one observes a continuous fall in pressure upon increase in volume until a minima is reached for very large values of inflation. This behaviour usually occurs in the presence of large magnetic fields and has been referred to as magnetic limit point earlier (Barham et al., 2007; Reddy and Saxena, 2017, 2018). As the strength of dipole is reduced $(\mathcal{M}=200)$ or its distance from membrane is increased $(\mathcal{M}=600, h=-3.5)$, the pressure-volume characteristics converge towards the purely mechanical case.

In each of the equilibria shown in Figures 4 and 5, we also compute the stability of the solution based on the criteria described in Section 3.3. It is observed that in all the cases the matrix $\mathbf{P}$ remains positive definite thus satisfying the necessary condition of stability. However, several equilibria do not satisfy the sufficient condition and these are denoted as dots in Figures 4 and 5 . We are unable to comment on the stability of these configurations based on our criteria. We plot a few membrane profiles corresponding to dipoles of strength $\mathcal{M}=200,400,600$ placed at a location $h=6.5$ in Figure 6. Typically the 'rounder' profiles with low $\eta(0)$ values tend to be stable and also have higher pressure values. Profiles with large $\eta(0)$ value and corresponding to small volumes and also low pressure are most likely unstable and fall in the regime of post-second limit point observed in the $P-V$ curves of Figure 4.

\subsubsection{Wrinkling behaviour}

Wrinkling of the membrane is associated with occurrence of compressive stresses in the plane of membrane as described in Section 3.4. A few membrane profiles for the cases $\mathcal{M}=100,200$ and $h=4$ 


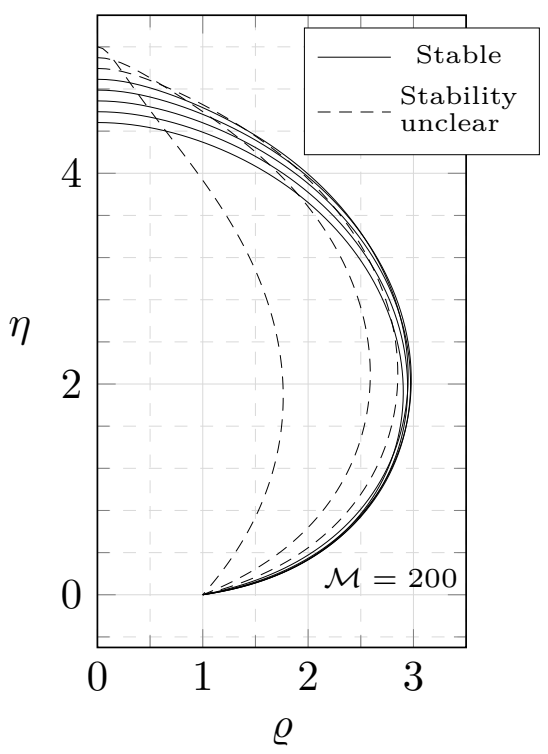

(a)

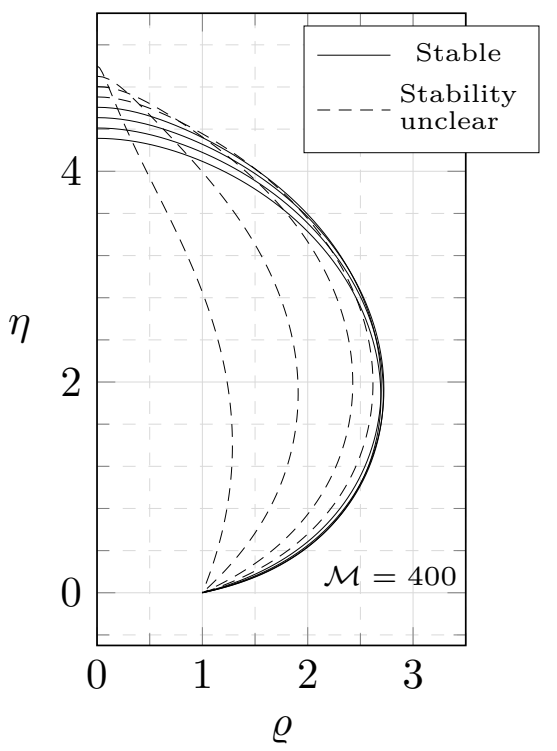

(b)

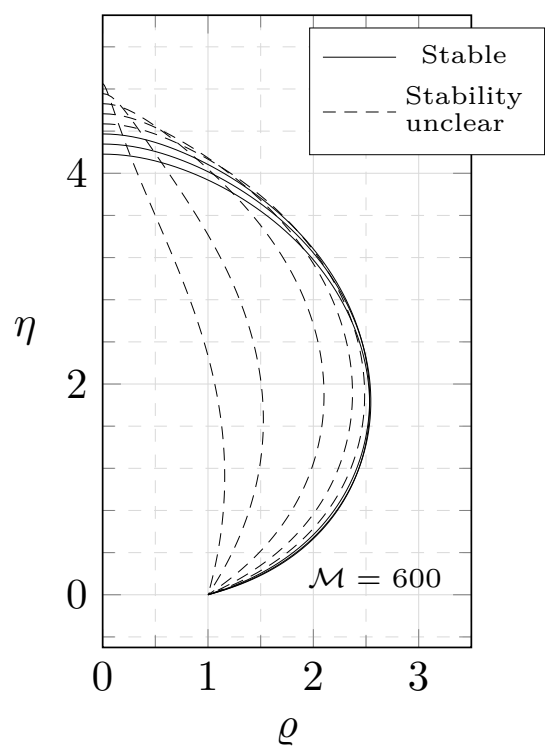

(c)

Figure 6: Membrane profiles for different pressure values and the location of magnet at $h=6.5$. (a) $\mathcal{M}=200,\{P=1.27,1.71,1.91,1.99,2.01,2.00,1.98,1.94\}$ for decreasing $\eta(0)$ value, (b) $\mathcal{M}=$ 400, $\{P=1.09,1.33,1.61,1.73,1.79,1.81,1.80,1.79\}$ for decreasing $\eta(0)$ value, (c) $\mathcal{M}=600,\{P=$ $1.04,1.18,1.42,1.57,1.65,1.679,1.688,1.683\}$ for decreasing $\eta(0)$ value.

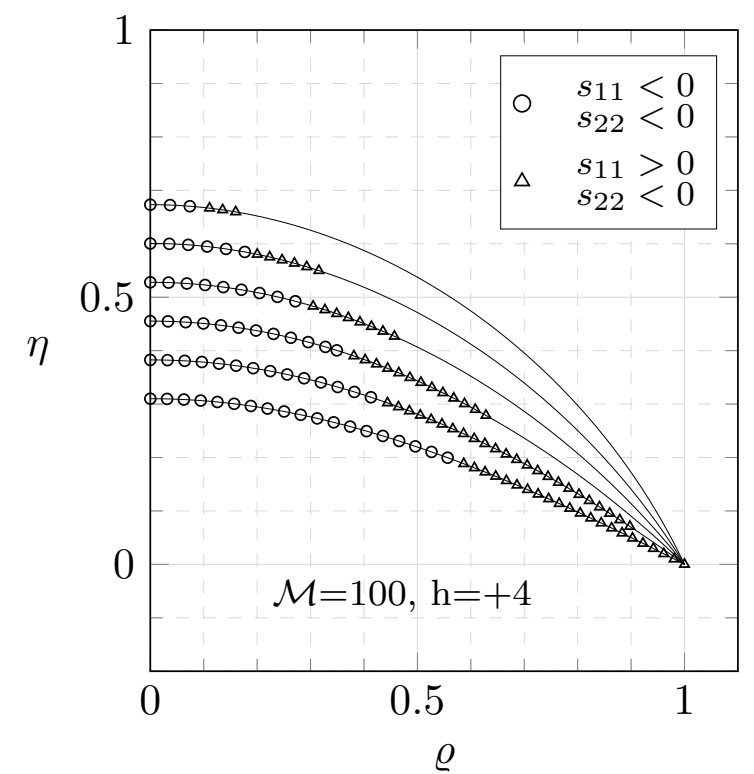

(a)

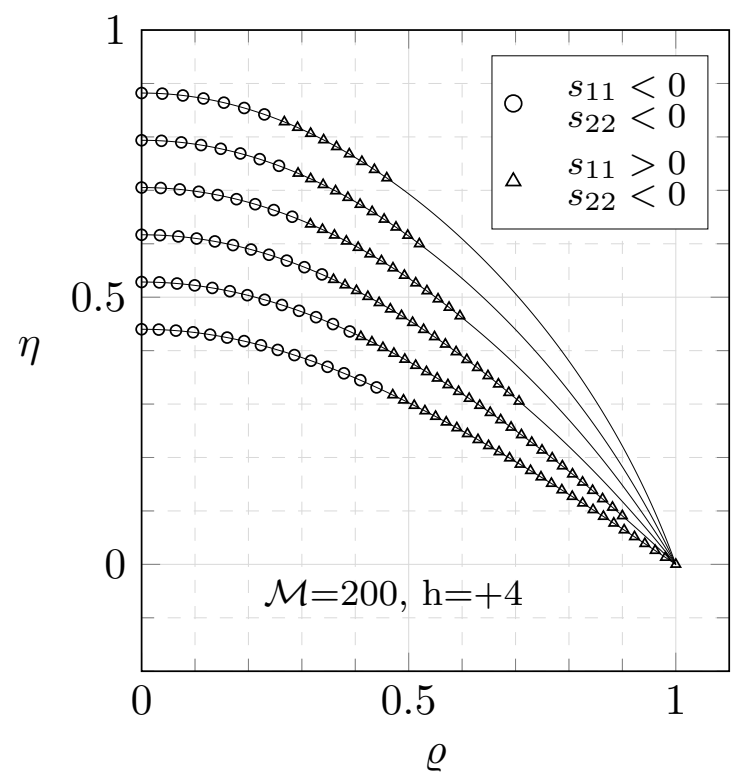

(b)

Figure 7: Membrane profiles when a dipole is placed above the membrane. Double wrinkling and wrinkling are both observed in the central region of the membrane. 


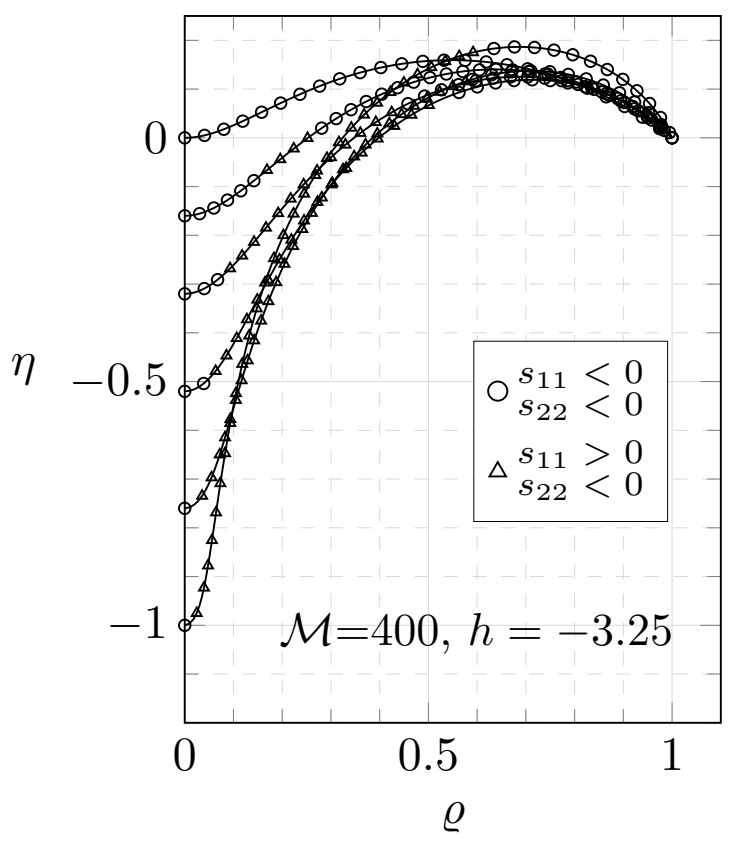

(a)

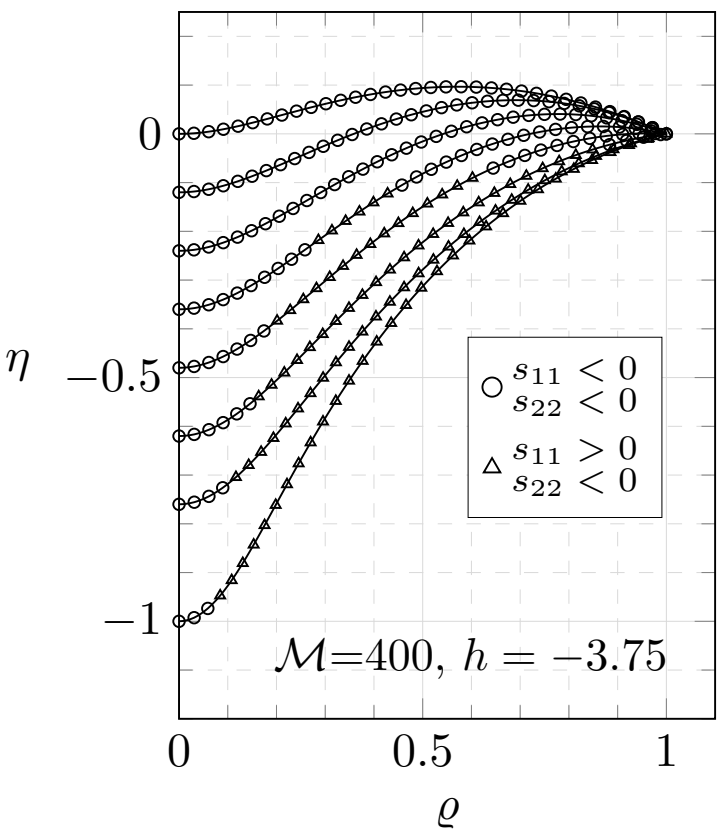

(b)

Figure 8: Membrane profiles when a dipole is placed below the membrane (low inflation). (a) $\mathcal{M}=$ $400, h=-3.25$, stability of all the six profiles is unclear. $\{P=2.23,2.49,2.79,3.16,3.51,3.72\}$ for decreasing $\eta(0)$ value. (b) $\mathcal{M}=400, h=-3.75$, Stability of top and bottom profiles is not clear while the other six profiles are stable. $\{P=0.95,1.04,1.14,1.23,1.288,1.29,1.287,1.44\}$ for decreasing $\eta(0)$ value.

are plotted in Figure 7. Triangles denote the region with $s_{22}<0$ representing wrinkles oriented along the radial direction while circles denote the region with $s_{11}<0, s_{22}<0$ representing double wrinkling. Wrinkling is prevalent in the central region of the membrane; since the loading is more uniform at the centre (both principal stretches being equal) there is a likelihood of the stresses $s_{11}$ and $s_{22}$ to be close to each other - resulting in double wrinkling. As we move further away from the centre, the non-uniformity in the stress values becomes more prominent and it is observed that the circumferential stress stays compressive leading to wrinkles spread in that direction. Increasing the volume leads to higher stretching and therefore a positive increase in the stress values, thereby resulting in a smaller wrinkled region. An increase in the dipole strength leads to an increase in the wrinkled region since the Maxwell stress tends to be compressive in the plane of membrane.

Note that all the cases shown in Figure 7 correspond to both the principal stretches $\lambda_{1}>1$ and $\lambda_{2}>1$. Wrinkles appear even though the membrane appears to be "stretched" because the total magnetoelastic Cauchy stresses are compressive. This observation is in agreement with results obtained by Otténio et al. (2008); Saxena and Ogden (2011) for surface instabilities in bulk magnetoelastic solids where instabilities arise in the presence of magnetic field even in the case of principal stretches being greater than one.

Profiles for the cases when the dipole is placed below the membrane are plotted in Figures 8 (low values of inflation) and 9 (large values of inflation). In the initial phase of low inflation, the membrane is pulled below towards the dipole and in the two cases seen $(h=-3.25,-3.75)$ entirety of the membrane has either single or double wrinkling. Profiles with small absolute value of $\eta(0)$ tend to have very large area with double wrinkling since in these cases elastic stresses are very small. As $|\eta(0)|$ increases, the area of double wrinkling is reduced and that of single wrinkling increases. Single wrinkles always occur when $s_{22}<0$ thereby causing wrinkles oriented along the radial direction.

For large inflation values cases presented in Figure 9, we observe that major part of the membrane is taut and wrinkles are created only near the outer edge. This is a marked reversal of behaviour in comparison to the cases in which dipole is placed above the membrane causing wrinkling primarily in 


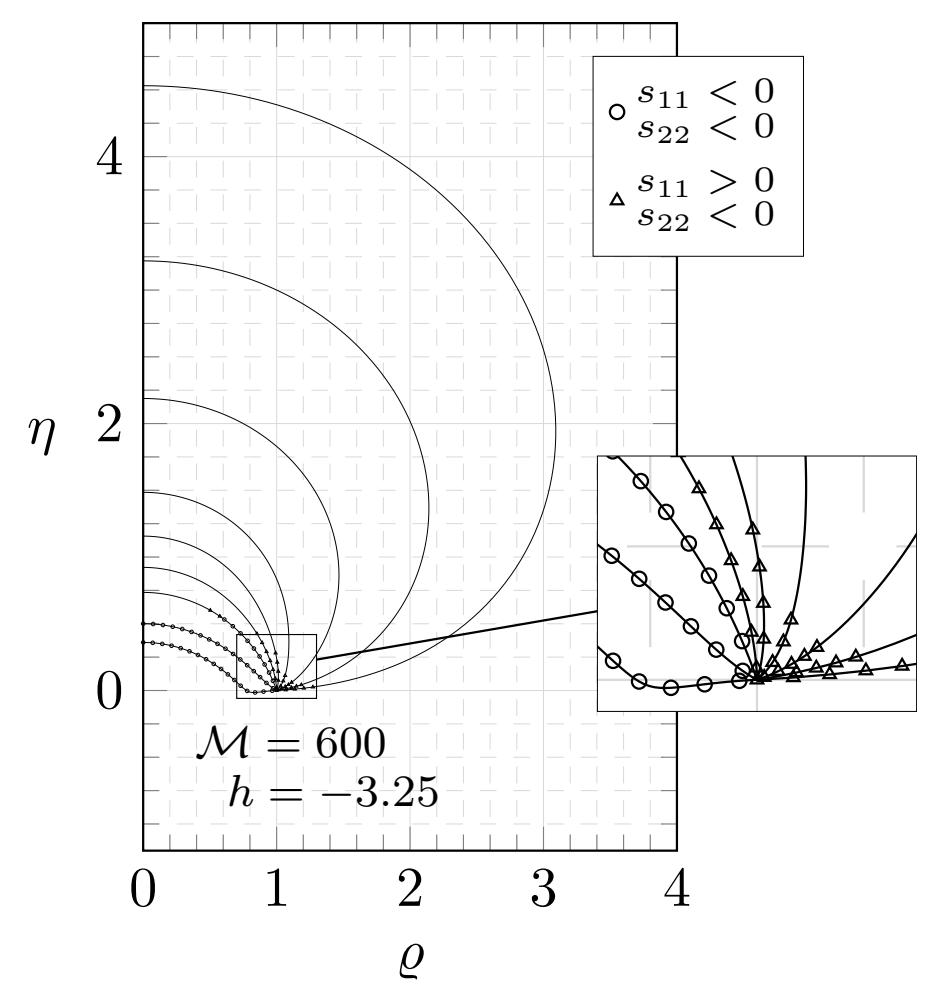

Figure 9: Membrane profiles when a dipole is placed below the membrane $(\mathcal{M}=600, h=-3.25)$ for large values of inflation. Wrinkling is observed near the ends $(r=1)$ where membrane is clamped. This is shown in the zoomed-in version. Stability of lower-most profile is uncertain; all other profiles are stable. $\{P=1.7,1.5,1.6,1.8,2.1,2.3,2.4,2.5,3.0\}$ for decreasing $\eta(0)$ value.

the central region.

Furthermore, an exhaustive analysis of wrinkling has also been carried out for all the cases studied in Figures 4 and 5. Every point on each of those curves represents a distinct profile of the membrane and the wrinkling status is described using symbols. It can be observed that membranes always have some wrinkled regions for all cases with $h<0$. Typically transition from double to single wrinkling occurs upon an increase of volume. For the cases with $h>0$, wrinkling happens for either very small or very large values of inflation - there is a significant region of loading conditions where the membrane inflates without any wrinkling.

The wrinkled profiles presented above are only approximate since they actually have regions with negative values of $s_{11}$ and $s_{22}$ and membranes can't sustain compressive stresses. An efficient way of tackling this issue in hyperelastic membranes is by introduction of a 'relaxed energy density function' using the tension field theory (Pipkin, 1986; Steigmann, 1990). Whenever the stretch value in a principal direction falls below the 'natural width in simple tension', the strain energy density function is replaced by a modified counterpart called the relaxed energy density. Calculations performed after incorporating this modification result in accurate membrane profiles. However, at present there is no extension of the tension field theory for magnetoelastic membranes. Results presented in this paper are a motivation to perform further research in this area for a more accurate prediction of membrane profiles and stress values.

\subsubsection{Multiple equilibria}

For a given set of pressure and magnetic loading conditions, we observe multiple stable solutions as demonstrated in Figure 10. For the case $h=-3.5$, all the profiles obtained are stable while for the case $h=6.5$, stability of three profiles is confirmed while stability of the fourth is not clear. These results corroborate the findings in recent papers (Reddy and Saxena, 2017, 2018) for toroidal and cylindrical membranes that multiple stable equilibrium configurations are attainable for thin magnetoelastic 


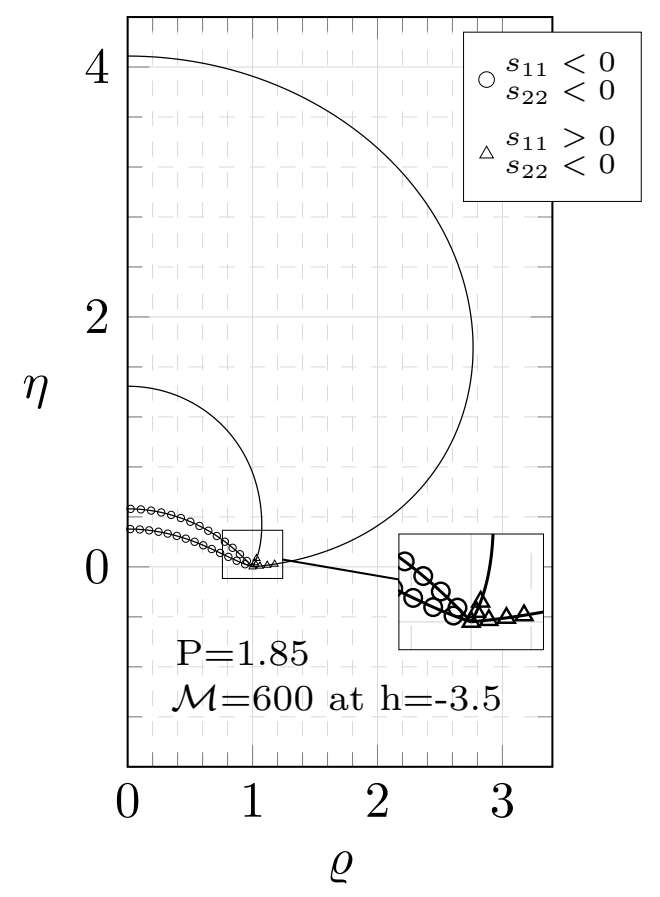

(a)

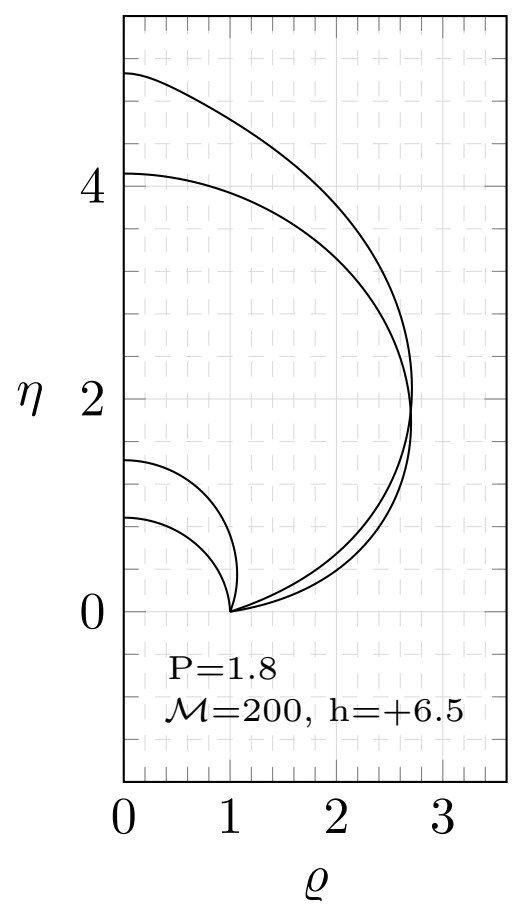

(b)

Figure 10: Membrane profiles for multiple equilibria obtained for a given set of pressure and magnetic loading conditions. (a) $P=1.85, \mathcal{M}=600, h=-3.5$. All profiles are stable and wrinkling is observed. (b) $P=1.8, \mathcal{M}=200, h=6.5$. Stability of profile with largest $\eta(0)$ is uncertain; the other three profiles are stable.

structures under coupled loading conditions. This implies that by keeping loading conditions $(P, \mathcal{M}$ and $h$ ) constant, one simply needs to perturb the membrane to oscillate among the various stable configurations.

\section{Conclusions}

Inflation of an isotropic incompressible circular magnetoelastic membrane in the presence of a magnetic dipole has been studied in this paper. The variational formulation is similar to that originally used by Barham et al. (2007, 2008) for a similar problem and then later by Reddy and Saxena (2017, 2018) to study various forms of instabilities in magnetoelastic membranes. Computations show instances of limit point and magnetic limit point for this problem similar to that obtained by Barham et al. We also demonstrate multiple stable and unstable equilibria under a given set of loading conditions for this problem - a result similar to that obtained for toroidal and cylindrical membranes by Reddy and Saxena in the papers mentioned above.

A key focus of this paper is on the determination of wrinkling instabilities due to magnetoelastic loading. We observe wrinkles due to development of compressive stresses in the central region of membrane when the dipole is placed in the direction of inflation and along the fixed sides when the dipole is placed opposite to the direction of inflation. Stronger magnetic field generated by either increasing the strength of dipole or by reducing the distance from the membrane results in a greater area being doubly wrinkled.

We also acknowledge the shortcomings of the present theory in the prediction of accurate profiles of membrane under magnetoelastic wrinkling due to a lack of extension of tension field theory to magnetoelasticity. Results in this paper provide a motivation for developments in this area and enquiry in this direction will be the focus of an upcoming research work.

Acknowledgements: Major part of this work was conducted when PS was based at IIT Hyderabad, 
India. This research was financially supported by a Ramanujan fellowship awarded to PS by the Science and Engineering Research Board.

\section{A Calculation of matrices $\mathbf{P}$ and $\mathrm{Q}$}

In this appendix, we list the several calculations required to calculate the entries in matrices $\mathbf{P}$ and $\mathbf{Q}$ used to assess stability in Section 3.3.

Using equation (14), we derive the following relations

$$
\begin{array}{r}
\lambda_{1, \varrho}=0, \quad \lambda_{1, \varrho^{\prime}}=\frac{\varrho^{\prime}}{\lambda_{1}}, \quad \lambda_{1, \eta}=0, \quad \lambda_{1, \eta^{\prime}}=\frac{\eta^{\prime}}{\lambda_{1}}, \\
\lambda_{2, \varrho}=\frac{1}{r}, \quad \lambda_{2, \varrho^{\prime}}=0, \quad \lambda_{2, \eta}=0, \quad \lambda_{2, \eta^{\prime}}=0, \\
\lambda_{1, \varrho^{\prime} \varrho^{\prime}}=\frac{1}{\lambda_{1}}-\frac{\varrho^{\prime 2}}{\lambda_{1}^{3}}, \quad \lambda_{1, \varrho^{\prime} \eta^{\prime}}=-\frac{\varrho^{\prime} \eta^{\prime}}{\lambda_{1}^{3}}, \quad \lambda_{1, \eta^{\prime} \eta^{\prime}}=\frac{1}{\lambda_{1}}-\frac{\eta^{\prime 2}}{\lambda_{1}^{3}}, \\
\lambda_{1}^{\prime}=\frac{\varrho^{\prime} \varrho^{\prime \prime}+\eta^{\prime} \eta^{\prime \prime}}{\lambda_{1}}, \quad \lambda_{2}^{\prime}=\frac{\varrho^{\prime}}{r}-\frac{\varrho}{r^{2}} .
\end{array}
$$

Upon using the relations (62) and applying chain rule of differentiation, we note

$$
\begin{array}{r}
\widehat{W}_{\varrho}=\widehat{W}_{\lambda_{2}} \lambda_{2, \varrho}, \quad \widehat{W}_{\varrho^{\prime}}=\widehat{W}_{\lambda_{1}} \lambda_{1, \varrho^{\prime}}, \quad \widehat{W}_{\eta}=0, \quad \widehat{W}_{\eta^{\prime}}=\widehat{W}_{\lambda_{1}} \lambda_{1, \eta^{\prime}}, \\
\widehat{W}_{\varrho \varrho}=\widehat{W}_{\lambda_{2} \lambda_{2}}\left[\lambda_{2, \varrho}\right]^{2}, \quad \widehat{W}_{\varrho^{\prime} \varrho^{\prime}}=\widehat{W}_{\lambda_{1}} \lambda_{1, \varrho^{\prime} \varrho^{\prime}}+\widehat{W}_{\lambda_{1} \lambda_{1}}\left[\lambda_{1, \varrho^{\prime}}\right]^{2}, \\
\widehat{W}_{\varrho^{\prime} \eta^{\prime}}=\widehat{W}_{\lambda_{1}} \lambda_{1, \varrho^{\prime} \eta^{\prime}}+\widehat{W}_{\lambda_{1} \lambda_{1}} \lambda_{1, \varrho^{\prime}} \lambda_{1, \eta^{\prime}}, \quad \widehat{W}_{\eta^{\prime} \eta^{\prime}}=\widehat{W}_{\lambda_{1}} \lambda_{1, \eta^{\prime} \eta^{\prime}}+\widehat{W}_{\lambda_{1} \lambda_{1}}\left[\lambda_{1, \eta^{\prime}}\right]^{2}, \\
\widehat{W}_{\varrho \varrho^{\prime}}=\widehat{W}_{\lambda_{1} \lambda_{2}} \lambda_{1, \varrho^{\prime}} \lambda_{2, \varrho}, \quad \widehat{W}_{\varrho \eta^{\prime}}=\widehat{W}_{\lambda_{1} \lambda_{2}} \lambda_{1, \eta^{\prime}} \lambda_{2, \varrho} .
\end{array}
$$

For the Ogden strain energy density given in equation (33), we note the following derivatives

$$
\begin{array}{r}
\widehat{W}_{\lambda_{1}}=\sum_{k=1}^{K} \mu_{k}\left[\lambda_{1}^{\alpha_{k}-1}-\frac{1}{\lambda_{1}^{\alpha_{k}+1} \lambda_{2}^{\alpha_{k}}}\right], \quad \widehat{W}_{\lambda_{2}}=\sum_{k=1}^{K} \mu_{k}\left[\lambda_{2}^{\alpha_{k}-1}-\frac{1}{\lambda_{2}^{\alpha_{k}+1} \lambda_{1}^{\alpha_{k}}}\right], \\
\widehat{W}_{\lambda_{1} \lambda_{1}}=\sum_{k=1}^{K} \mu_{k}\left[\left[\alpha_{k}-1\right] \lambda_{1}^{\alpha_{k}-2}+\frac{\alpha_{k}+1}{\lambda_{1}^{\alpha_{k}+2} \lambda_{2}^{\alpha_{k}}}\right], \quad \widehat{W}_{\lambda_{1} \lambda_{2}}=\sum_{k=1}^{K} \frac{\alpha_{k} \mu_{k}}{\left[\lambda_{1} \lambda_{2}\right]^{\alpha_{k}+1}}, \\
\widehat{W}_{\lambda_{2} \lambda_{2}}=\sum_{k=1}^{K} \mu_{k}\left[\left[\alpha_{k}-1\right] \lambda_{2}^{\alpha_{k}-2}+\frac{\alpha_{k}+1}{\lambda_{1}^{\alpha_{k}} \lambda_{2}^{\alpha_{k}+2}}\right] .
\end{array}
$$

Given the integrand $\mathcal{F}$ from equation (41) the components of matrix $\mathbf{P}$ as shown in equation (44a) are given by

$$
P_{1}=T R_{0}^{2} r \widehat{W}_{\varrho^{\prime} \varrho^{\prime}}, \quad P_{2}=P_{3}=T R_{0}^{2} r \widehat{W}_{\varrho^{\prime} \eta^{\prime}}, \quad P_{4}=T R_{0}^{2} r \widehat{W}_{\eta^{\prime} \eta^{\prime}},
$$

where the derivatives of energy $\widehat{W}$ are calculated above.

Let $L=R_{0}^{6}\left|\boldsymbol{h}_{a}\right|^{2} / D^{2}$, then upon using the non-dimensional entities $\mathcal{M}$ and $P$ defined in equations (37) and (38), we can rewrite the integrand $\mathcal{F}$ from equation (41) as

$$
\mathcal{F}=\mu R_{0}^{2} \operatorname{Tr}\left[\frac{\widehat{W}}{\mu}-\frac{1}{2} \chi \mathcal{M} L+\frac{P}{3 r}\left[\varrho^{2} \eta^{\prime}-\varrho \varrho^{\prime} \eta\right]\right] .
$$

Non-zero second derivatives of the quantity $L$ are calculated as

$$
L_{\varrho \varrho}=\frac{6\left[7 \varrho^{4}+42 \varrho^{2}[\eta-h]^{2}-5[\eta-h]^{4}\right]}{\left[\varrho^{2}+[\eta-h]^{2}\right]^{6}}, \quad L_{\varrho \eta}=\frac{240 \varrho[\eta-h]^{3}}{\left[\varrho^{2}+[\eta-h]^{2}\right]^{6}},
$$




$$
L_{\eta \eta}=\frac{-24[\eta-h]^{2}\left[3 \varrho^{2}-7[\eta-h]^{2}\right]}{\left[\varrho^{2}+[\eta-h]^{2}\right]^{6}}
$$

Let the matrix $\mathbf{Q}$ be denoted as

$$
\mathbf{Q}=\frac{1}{2}\left[\mathbf{R}-\frac{d}{d r} \mathbf{S}\right]
$$

where $\mathbf{R}$ and $\mathbf{S}$ are matrices of derivatives of $\mathcal{F}$ from equation (43). Derivatives of $\mathcal{F}$ can be taken to populate the matrix $\mathbf{R}$ as follows

$$
\mathbf{R}=\left[\begin{array}{cc}
\mathcal{F}_{\varrho \varrho} & \mathcal{F}_{\varrho \eta} \\
\mathcal{F}_{\varrho \eta} & \mathcal{F}_{\eta \eta}
\end{array}\right]=\mu R_{0}^{2} \operatorname{Tr}\left[\begin{array}{cc}
\frac{1}{\mu} \widehat{W}_{\varrho \varrho}-\frac{1}{2} \chi \mathcal{M} L_{\varrho \varrho}+\frac{2 \eta^{\prime} P}{3 r} & -\frac{\chi \mathcal{M} L_{\varrho \eta}}{2}-\frac{\varrho^{\prime} P}{3 r} \\
-\frac{\chi \mathcal{M} L_{\varrho \eta}}{2}-\frac{\varrho^{\prime} P}{3 r} & -\frac{\chi \mathcal{M} L_{\eta \eta}}{2}
\end{array}\right]
$$

while the matrix $\mathbf{S}$ is given as

$$
\mathbf{S}=\left[\begin{array}{cc}
\mathcal{F}_{\varrho \varrho^{\prime}} & \mathcal{F}_{\varrho \eta^{\prime}} \\
\mathcal{F}_{\varrho^{\prime} \eta} & \mathcal{F}_{\eta \eta^{\prime}}
\end{array}\right]=\mu R_{0}^{2} \operatorname{Tr}\left[\begin{array}{cc}
\frac{1}{\mu} \widehat{W}_{\varrho \varrho^{\prime}}-\frac{\eta P}{3 r} & \frac{1}{\mu} \widehat{W}_{\varrho \eta^{\prime}}+\frac{2 \varrho P}{3 r} \\
-\frac{\varrho P}{3 r} & 0
\end{array}\right]
$$

Diffrentiating $\mathbf{S}$ gives

$$
\frac{d \mathbf{S}}{d r}=R_{0}^{2} T\left[\begin{array}{cc}
\frac{\varrho^{\prime \prime}}{\lambda_{1}} \widehat{W}_{\lambda_{1} \lambda_{2}}+\varrho^{\prime} \gamma-\frac{\mu \eta^{\prime} P}{3} & \frac{\eta^{\prime \prime}}{\lambda_{1}} \widehat{W}_{\lambda_{1} \lambda_{2}}+\eta^{\prime} \gamma+\frac{2 \mu \varrho^{\prime} P}{3} \\
-\frac{\mu \varrho^{\prime} P}{3} & 0
\end{array}\right],
$$

where for the case of Ogden energy density $\gamma$ is given as

$$
\gamma=\frac{d}{d r}\left(\frac{\widehat{W}_{\lambda_{1} \lambda_{2}}}{\lambda_{1}}\right)=-\frac{\varrho^{\prime} \varrho^{\prime \prime}+\eta^{\prime} \eta^{\prime \prime}}{\lambda_{1}} \sum_{k=1}^{K} \frac{\mu_{k} \alpha_{k}\left[\alpha_{k}+2\right]}{\lambda_{1}^{\alpha_{k}+3} \lambda_{2}^{\alpha_{k}+1}}-\frac{\varrho^{\prime} r-\varrho}{r^{2}} \sum_{k=1}^{K} \frac{\mu_{k} \alpha_{k}\left[\alpha_{k}+1\right]}{\left[\lambda_{1} \lambda_{2}\right]^{\alpha_{k}+2}}
$$

Thus, we have the following components of $\boldsymbol{Q}$ as per the entries in equation (44a)

$$
\begin{aligned}
& Q_{1}=\mu R_{0}^{2} \operatorname{Tr}\left[\frac{1}{\mu} \widehat{W}_{\varrho \varrho}-\frac{1}{2} \chi \mathcal{M} L_{\varrho \varrho}+\frac{2 \eta^{\prime} P}{3 r}\right]-R_{0}^{2} T\left[\frac{\varrho^{\prime \prime}}{\lambda_{1}} \widehat{W}_{\lambda_{1} \lambda_{2}}+\varrho^{\prime} \gamma-\frac{\mu \eta^{\prime} P}{3}\right] \\
& Q_{2}=-\mu R_{0}^{2} \operatorname{Tr}\left[\frac{\chi \mathcal{M} L_{\varrho \eta}}{2}+\frac{\varrho^{\prime} P}{3 r}\right]-R_{0}^{2} T\left[\frac{\eta^{\prime \prime}}{\lambda_{1}} \widehat{W}_{\lambda_{1} \lambda_{2}}+\eta^{\prime} \gamma+\frac{2 \mu \varrho^{\prime} P}{3}\right] \\
& Q_{3}=-\mu R_{0}^{2} \operatorname{Tr}\left[\frac{\chi \mathcal{M} L_{\varrho \eta}}{2}+\frac{\varrho^{\prime} P}{3 r}\right]+R_{0}^{2} T \frac{\mu \varrho^{\prime} P}{3} \\
& Q_{4}=-\mu R_{0}^{2} \operatorname{Tr} \frac{\chi \mathcal{M} L_{\eta \eta}}{2}
\end{aligned}
$$

\section{B Bibliography}

Barham M., Steigmann D.J., McElfresh M., and Rudd R.E. "Finite deformation of a pressurized magnetoelastic membrane in a stationary dipole field". Acta Mechanica, 191(1-2):1-19 (2007)

Barham M., Steigmann D.J., McElfresh M., and Rudd R.E. "Limit-point instability of a magnetoelastic membrane in a stationary magnetic field". Smart Materials and Structures, 17(5):055003 (2008)

Barham M.I., White D.A., and Steigmann D.J. "Finite element modeling of the deformation of magnetoelastic film". Journal of Computational Physics, 229(18):6193-6207 (2010) 
Böse H., Rabindranath R., and Ehrlich J. "Soft magnetorheological elastomers as new actuators for valves". Journal of Intelligent Material Systems and Structures, page 1045389X11433498 (2012)

Böse H. and Röder R. "Magnetorheological elastomers with high variability of their mechanical properties". In "Journal of physics: Conference Series", volume 149, page 012090. IOP Publishing (2009)

Brown W.F. Magnetoelastic interactions. Springer, New York (1966)

Bustamante R. "Transversely isotropic nonlinear magneto-active elastomers". Acta Mechanica, 210(34):183-214 (2010)

Castañeda P.P. and Galipeau E. "Homogenization-based constitutive models for magnetorheological elastomers at finite strain". Journal of the Mechanics and Physics of Solids, 59(2):194-215 (2011)

Castaneda P.P. and Siboni M. "A finite-strain constitutive theory for electro-active polymer composites via homogenization". International Journal of Non-Linear Mechanics, 47(2):293-306 (2012)

Chatzigeorgiou G., Javili A., and Steinmann P. "Unified magnetomechanical homogenization framework with application to magnetorheological elastomers". Mathematics and Mechanics of Solids, 19(2):193211 (2014)

Danas K., Kankanala S.V., and Triantafyllidis N. "Experiments and modeling of iron-particle-filled magnetorheological elastomers". Journal of the Mechanics and Physics of Solids, 60:120-138 (2012)

Danas K. and Triantafyllidis N. "Instability of a magnetoelastic layer resting on a non-magnetic substrate". Journal of the Mechanics and Physics of Solids, 69(1):67-83 (2014)

Dorfmann A. and Ogden R.W. "Magnetoelastic modelling of elastomers". European Journal of Mechanics- A/Solids, 22(4):497-507 (2003)

Dorfmann L. and Ogden R.W. Nonlinear theory of electroelastic and magnetoelastic interactions. Springer, New York (2014)

Ethiraj G. and Miehe C. "Multiplicative magneto-elasticity of magnetosensitive polymers incorporating micromechanically-based network kernels". International Journal of Engineering Science, 102:93 119 (2016)

Gelfand I.M. and Fomin S.V. Calculus of Variations,(Translated and edited by Silverman, RA). Dover Edition (2000)

Ginder J.M., Clark S.M., Schlotter W.F., and Nichols M.E. "Magnetostrictive phenomena in magnetorheological elastomers". International Journal of Modern Physics B, 16(17n18):2412-2418 (2002)

Ginder J.M., Schlotter W.F., and Nichols M.E. "Magnetorheological elastomers in tunable vibration absorbers". In "SPIE's 8th Annual International Symposium on Smart Structures and Materials", pages 103-110. International Society for Optics and Photonics (2001)

Goshkoderia A. and Rudykh S. "Stability of magnetoactive composites with periodic microstructures undergoing finite strains in the presence of a magnetic field". Composites Part B: Engineering, 128:19 $-29(2017)$

Grossman G. "Analysis of rim supports for off-axis inflatable reflectors. i: Loads". Journal of Aerospace Engineering, 4(1):47-66 (1991a)

Grossman G. "Analysis of rim supports for off-axis inflatable reflectors. ii: Deformations". Journal of Aerospace Engineering, 4(1):67-77 (1991b) 
Haldar K., Kiefer B., and Menzel A. "Finite element simulation of rate-dependent magneto-active polymer response". Smart Materials and Structures, 25(10):104003 (2016)

Hu W., Lum G.Z., Mastrangeli M., and Sitti M. "Small-scale soft-bodied robot with multimodal locomotion". Nature, 554:81-85 (2018)

Jolly M.R., Carlson J.D., and Munoz B.C. "A model of the behaviour of magnetorheological materials". Smart Materials and Structures, 5(5):607 (1996)

Kankanala S.V. and Triantafyllidis N. "On finitely strained magnetorheological elastomers". Journal of the Mechanics and Physics of Solids, 52(12):2869-2908 (2004)

Kankanala S.V. and Triantafyllidis N. "Magnetoelastic buckling of a rectangular block in plane strain". Journal of the Mechanics and Physics of Solids, 56(4):1147-1169 (2008)

Keplinger C., Li T., Baumgartner R., Suo Z., and Bauer S. "Harnessing snap-through instability in soft dielectrics to achieve giant voltage-triggered deformation". Soft Matter, 8(2):285-288 (2012)

Khayat R.E. and Derdouri A. "Inflation of hyperelastic cylindrical membranes as applied to blow moulding. part i. axisymmetric case". International Journal for Numerical Methods in Engineering, 37(22):3773-3791 (1994a)

Khayat R.E. and Derdouri A. "Inflation of hyperelastic cylindrical membranes as applied to blow moulding. part ii. non-axisymmetric case". International Journal for Numerical Methods in Engineering, 37(22):3793-3808 (1994b)

Leone J.E. "Infusion balloon catheter" (1994). US Patent 5,318,531

Li T., Keplinger C., Baumgartner R., Bauer S., Yang W., and Suo Z. "Giant voltage-induced deformation in dielectric elastomers near the verge of snap-through instability". Journal of the Mechanics and Physics of Solids, 61(2):611-628 (2013)

Liang X. and Cai S. "New electromechanical instability modes in dielectric elastomer balloons". International Journal of Solids and Structures, pages 1-9 (2017)

Maugin G.A. and Eringen A.C. "Deformable magnetically saturated media. i. field equations". Journal of Mathematical Physics, 13(2):143-155 (1972)

Mayer M., Rabindranath R., Börner J., Hörner E., Bentz A., Salgado J., Han H., Böse H., Probst J., Shamonin M., Monkman G.J., and Schlunck G. "Ultra-Soft PDMS-Based Magnetoactive Elastomers as Dynamic Cell Culture Substrata." PloS one, 8(10):e76196 (2013)

Ogden R.W. "Large deformation isotropic elasticity - on the correlation of theory and experiment for incompressible rubberlike solids". In "Proceedings of the Royal Society of London A: Mathematical, Physical and Engineering Sciences", volume 326, pages 565-584 (1972)

Otténio M., Destrade M., and Ogden R.W. "Incremental magnetoelastic deformations, with application to surface instability". Journal of Elasticity, 90(1):19-42 (2008)

Pao Y.H. "Electromagnetic forces in deformable continua". In "Mechanics today. Volume 4.(A78-35706 14-70) New York, Pergamon Press, Inc., 1978, p. 209-305. NSF-supported research.", volume 4, pages 209-305 (1978)

Patil A. and Dasgupta A. "Finite inflation of an initially stretched hyperelastic circular membrane". European Journal of Mechanics / A Solids, 41:28-36 (2013)

Pipkin A.C. "The relaxed energy density for isotropic elastic membranes". IMA Journal of Applied Mathematics, 36(1):85-99 (1986) 
Raikher Y.L., Stolbov O.V., and Stepanov G.V. "Deformation of a circular ferroelastic membrane in a uniform magnetic field". Technical Physics, 53(9):1169-1176 (2008)

Reddy N.H. and Saxena P. "Limit points in the free inflation of a magnetoelastic toroidal membrane". International Journal of Non-Linear Mechanics, 95(C):248-263 (2017)

Reddy N.H. and Saxena P. "Instabilities in the axisymmetric magnetoelastic deformation of a cylindrical membrane". International Journal of Solids and Structures, 136-137:203-219 (2018)

Rudykh S. and Bertoldi K. "Stability of anisotropic magnetorheological elastomers in finite deformations: A micromechanical approach". Journal of the Mechanics and Physics of Solids, 61(4):949-967 (2013)

Rudykh S., Bhattacharya K., and deBotton G. "Snap-through actuation of thick-wall electroactive balloons". International Journal of Non-Linear Mechanics, 47(2):206 - 209 (2012). Nonlinear Continuum Theories

Saxena P. "Finite deformations and incremental axisymmetric motions of a magnetoelastic tube". Mathematics and Mechanics of Solids (2017)

Saxena P., Hossain M., and Steinmann P. "A theory of finite deformation magneto-viscoelasticity". International Journal of Solids and Structures, 50(24):3886-3897 (2013)

Saxena P., Hossain M., and Steinmann P. "Nonlinear magneto-viscoelasticity of transversally isotropic magneto-active polymers". Proceedings of the Royal Society A, 470(2166):20140082 (2014)

Saxena P. and Ogden R.W. "On surface waves in a finitely deformed magnetoelastic half-space". International Journal of Applied Mechanics, 03(04):633-665 (2011)

Saxena P., Pelteret J.P., and Steinmann P. "Modelling of iron-filled magneto-active polymers with a dispersed chain-like microstructure". European Journal of Mechanics - A/Solids, 50:132-151 (2015)

Steigmann D. "Tension-field theory". In "Proceedings of the Royal Society A, 429", pages 141-173 (1990)

Steigmann D.J. "Equilibrium theory for magnetic elastomers and magnetoelastic membranes". International Journal of Non-Linear Mechanics, 39(7):1193-1216 (2004)

Tielking J.T. "Analytic tire model. Phase I: The statically loaded toroidal membrane". Technical report, Transportation Research Institute (UMTRI) (1975)

Tiersten H. "Coupled magnetomechanical equations for magnetically saturated insulators". Journal of Mathematical Physics, 5(9):1298-1318 (1964)

Truesdell C. and Toupin R. "The classical field theories. in S. Flügge (ed.), Encyclopedia of physics. principles of classical mechanics and field theory (vol. iii/i)". pages 226-793 (1960)

Verron E. and Marckmann G. "Inflation of elastomeric circular membranes using network constitutive equations". International Journal of Non-Linear Mechanics, 38(8):1221-1235 (2003) 